\title{
LOEWNER THEORY IN ANNULUS I: EVOLUTION FAMILIES AND DIFFERENTIAL EQUATIONS
}

\author{
MANUEL D. CONTRERAS, SANTIAGO DÍAZ-MADRIGAL, AND PAVEL GUMENYUK
}

\begin{abstract}
Loewner theory, based on dynamical viewpoint, is a powerful tool in complex analysis, which plays a crucial role in such important achievements as the proof of the famous Bieberbach conjecture and the well-celebrated Schramm stochastic Loewner evolution (SLE). Recently, Bracci et al. proposed a new approach bringing together all the variants of the (deterministic) Loewner evolution in a simply connected reference domain. We construct an analog of this theory for the annulus. In this paper, the first of two articles, we introduce a general notion of an evolution family over a system of annuli and prove that there is a one-to-one correspondence between such families and semicomplete weak holomorphic vector fields. Moreover, in the non-degenerate case, we establish a constructive characterization of these vector fields analogous to the non-autonomous Berkson-Porta representation of Herglotz vector fields in the unit disk.
\end{abstract}

\section{Contents}

1. Introduction

1.1. Loewner theory

1.2. Statement of the problem and main results

2. Holomorphic Carathéodory differential equations

2.1. Weak holomorphic vector fields and Carathéodory ODEs

2.2. Semicomplete weak holomorphic vector fields and families of holomorphic functions generated by them

3. Evolution families in simply connected domains

4. Evolution families over systems of doubly connected domains

4.1. Definition of an evolution family in the doubly connected case

4.2. Lifting evolution families to a simply connected domain

4.3. Some auxiliary statements

Received by the editors November 18, 2010 and, in revised form, September 8, 2011.

2010 Mathematics Subject Classification. Primary 30C35, 30C20, 30D05; Secondary 30C80, $34 \mathrm{M} 15$.

Key words and phrases. Univalent functions, annulus, Loewner chains, Loewner evolution, evolution family, parametric representation.

The first and second authors were partially supported by the Ministerio de Ciencia e Innovación and the European Union (FEDER), project MTM2009-14694-C02-02.

The authors were partially supported by the ESF Networking Programme "Harmonic and Complex Analysis and its Applications" and by La Consejería de Economía, Innovación y Ciencia de la Junta de Andalucía (research group FQM-133).

The third author was supported by a grant from Iceland, Liechtenstein, and Norway through the EEA Financial Mechanism. Supported and coordinated by Universidad Complutense de Madrid and by Instituto de Matemáticas de la Universidad de Sevilla. Partially supported by the Scandinavian Network "Analysis and Applications" (NordForsk), project \#080151, and the Research Council of Norway, project \#177355/V30. 
4.4. Proofs

5. Evolution families and differential equations

5.1. Semicomplete weak holomorphic vector fields in non-degenerate case

5.2. Degenerate case

6. Examples

7. Comments on parametric representation of slit mappings

References

\section{INTRODUCTION}

1.1. Loewner theory. In recent years, there has been a dramatic growth of interest in the dynamical aspects in complex analysis. Most of all, this applies to the so-called Loewner theory, dealing with the semigroup $\mathrm{Hol}(\mathbb{D}, \mathbb{D})$ of all holomorphic self-maps of the unit disk $\mathbb{D}:=\{z:|z|<1\}$.

The core of modern Loewner theory resides in the connection and interplay among the following three basic notions:

- Herglotz vector fields $G: \mathbb{D} \times[0,+\infty) \rightarrow \mathbb{C}$, which are semicomplete time-dependent holomorphic vector fields in the unit disk $\mathbb{D}$ and can be described as integrable families $\left(G_{t}\right)_{t \geq 0}$ of infinitesimal generators of one-parametric semigroups in $\operatorname{Hol}(\mathbb{D}, \mathbb{D})$;

- Evolution families $\left(\varphi_{s, t}\right)_{t \geq s \geq 0}$, which can be characterized as nonautonomous holomorphic semiflows generated by Herglotz vector fields;

- Loewner chains $\left(f_{t}\right)_{t \geq 0}$, which are one-parametric families of univalent functions $f_{t}: \mathbb{D} \rightarrow \mathbb{C}$ with expanding systems of image domains $f_{t}(\mathbb{D})$. Any Loewner chain satisfies a linear PDE, known as (generalized) Loewner-Kufarev PDE, driven by a Herglotz vector field. The corresponding evolution family $\varphi_{s, t}:=f_{t}^{-1} \circ f_{s}$ can be obtained by solving the characteristic equation for this $\mathrm{PDE}$.

There is an essentially one-to-one correspondence between Herglotz vector fields, evolution families, and Loewner chains.

Classical Loewner theory originated from the so-called parametric representation of univalent functions proposed in 1923 by C. Loewner [35] and developed further by a number of specialists in geometric function theory, among which we would like to mention the fundamental contributions of Kufarev [28] and Pommerenke [37, 38, Ch. 6]. In the case that they considered, and which is usually referred to in modern literature as the (classical) radial case, a Loewner chain is a family of univalent functions $f_{t}(z)=e^{t} z+a_{2}(t) z^{2}+\ldots, z \in \mathbb{D}$, such that $f_{s}(\mathbb{D}) \subset f_{t}(\mathbb{D})$ whenever $t \geq s$. A (classical radial) Herglotz vector field is of the form $G(w, t):=-w p(w, t)$, where $p$ is holomorphic in $w \in \mathbb{D}$, measurable in $t \geq 0$, and satisfies conditions $p(0, t)=1$ and $\operatorname{Re} p(w, t)>0$ for all $w \in \mathbb{D}$ and a.e. $t \geq 0$. For any holomorphic vector field of this form there exists a unique classical radial Loewner chain $\left(f_{t}\right)$ such that $[s,+\infty) \ni t \mapsto \varphi_{s, t}(z):=f_{t}^{-1} \circ f_{s}(z)$ solves, for any $z \in \mathbb{D}$ and $s \geq 0$, the Loewner-Kufarev ODE $\dot{w}=G(w, t)$ with the initial condition $\left.w(t)\right|_{t=s}=z$. The converse is also true: any classical radial Loewner chain is a solution to the Loewner-Kufarev PDE $\partial f_{t}(z) / \partial t=-f^{\prime}(z) G(z, t)$ driven by some classical radial 
Herglotz vector field $G$, while the evolution family $\left(\varphi_{s, t}\right)$ corresponding to $\left(f_{t}\right)$ solves the Loewner-Kufarev ODE. Moreover, the Loewner chain $\left(f_{t}\right)$ can be reconstructed via its evolution family $\left(\varphi_{s, t}\right)$ by means of the formula $f_{s}=\lim _{t \rightarrow+\infty} e^{t} \varphi_{s, t}$.

This relation among $G,\left(f_{t}\right)$, and $\left(\varphi_{s, t}\right)$ provides a representation of the class $\mathcal{S}$ of all normalized holomorphic univalent functions in $\mathbb{D}$, since any $f_{0} \in \mathcal{S}$ can be embedded as an initial element into a classical radial Loewner chain [38, Th. 6.5 on p.159], 22. This representation was one of the main tools in the proof of the famous Bieberbach conjecture, given by de Branges [13] (see [20] for a simplified proof).

A similar representation, designated in modern terminology by the attribute chordal, was proposed by Kufarev and his students for holomorphic univalent selfmaps of the upper half-plane with the hydrodynamic normalization at the point of infinity [29]; see also references cited in [17, pp. 543-544].

Komatu [25] was the first who was able to apply Loewner's ideas for parametric representation of univalent functions in the annulus. His approach was developed by Goluzin [21, Li En Pir [34, Lebedev [33, and Gutljanskiǔ 23]. More general cases of Loewner evolution in a multiply connected context were studied in [27] and by an essentially different method in [31, 42. The monograph [4] contains a self-contained detailed account on the parametric representation both in simply and multiply connected cases.

Until the last decade the attention of specialists in Loewner theory was mainly paid to the radial case in the unit disk, first of all because of its applications in the study of the class $\mathcal{S}$ and its subclasses. The significance of the chordal Loewner evolution as well as the Loewner evolution in multiply connected domains was apparently underestimated. However, nowadays the parametric method, invented by Loewner to study the Bieberbach conjecture, has gone far beyond the scope of the original problem, and the distribution of active interest in various aspects of the theory has been changed. The most spectacular evidence of this fact is the wellcelebrated Stochastic Loewner evolution (SLE) invented in 2000 by Schramm [40. It appears that the chordal Loewner-Kufarev equation driven by Brownian motion is intrinsically related to several important lattice models in statistical physics, such as the critical Ising model. We refer interested readers to [32. For a wider discussion on connections of deterministic and stochastic Loewner evolutions to conformal field theory and integrable systems, see [36] and references cited therein.

To conclude, we would like to mention the survey paper [2] that covers the basics of classical and modern Loewner theory, its history, recent development, and applications.

1.2. Statement of the problem and main results. Recently Bracci and the first two authors of this paper [10, 11] introduced a new general approach in Loewner theory in the unit disk, which unifies, and contains as very special cases, both chordal and radial Loewner evolutions. This approach is based on a general intrinsic notion of evolution family in $\mathbb{D}$ (see Definition 3.1), which can be viewed as a nonautonomous generalization of continuous one-parametric semigroups in $\operatorname{Hol}(\mathbb{D}, \mathbb{D})$. Further developments in this direction can be found in [12, 14, 16, 17]. In this paper we address the following

Problem: Construct a general Loewner theory in the annulus. 
Our motivation is based on two reasons. First, although this abstract approach was generalized to arbitrary finite-dimensional complete hyperbolic complex manifolds [5, 11, applying it directly to any multiply connected hyperbolic Riemann surface $D$ which is not conformally equivalent to the punctured disk $\mathbb{D}^{*}:=\mathbb{D} \backslash\{0\}$ would lead to a quite trivial theory, because the connected component of $\operatorname{Hol}(D, D)$ containing $\mathrm{id}_{D}$ coincides with the group of rotations if $D$ is doubly connected, or consists of $\mathrm{id}_{D}$ only otherwise (see, e.g., 1 , §1.2.2]). It follows that in order to develop an interesting substantial theory for the multiply connected case, instead of a static reference domain or Riemann surface $D$, one has to consider a one-parametric family $\left(D_{t}\right)_{t \geq 0}$ of reference domains admitting existence of injective holomorphic mappings $\varphi_{s, t}: D_{s} \rightarrow D_{t}$ homotopically equivalent to the identity for any $s \geq 0$ and any $t \geq s$. In a doubly connected case it leads to a family of expanding annuli $D_{t}=\mathbb{A}_{r(t)}:=\{z: r(t)<|z|<1\}$, where $r:[0,+\infty) \rightarrow[0,1)$ is non-increasing and continuous. The first who implemented this idea was Komatu [25, but to the best of our knowledge all studies published of Loewner evolution in doubly and, more generally, multiply connected domains deal only with some special cases.

The other reason is the recent boost of interest in Loewner theory as a whole as well as to its variants for multiply connected domains. As an illustration, we cite papers [6] - 8], 45] extending the notion of SLE to the multiply connected case.

The study we present here is intended to be the first of two papers on the problem stated above; it contains the theory of evolution families and semicomplete weak holomorphic vector fields 1 while Loewner chains will be considered in another paper.

In Section 4 we introduce a notion of evolution family over a canonical domain system of annuli (see Definitions 4.1 and 4.3), analogous in a certain sense to that proposed in [10] for the unit disk, and we establish some basic properties of these evolution families. A key result is the following lifting theorem:

Theorem 4.6. Let $\left(D_{t}\right)=\left(\mathbb{A}_{r(t)}\right)$, be a non-degenerate canonical domain system of order $d \in[1,+\infty]$. Then for any $L^{d}$-evolution family $\left(\varphi_{s, t}\right)$ over $\left(D_{t}\right)$ there exists a unique $L^{d}$-evolution family $\left(\Psi_{s, t}\right)$ in the strip $\mathbb{S}:=\{z: 0<\operatorname{Re} z<1\}$ such that

$$
W_{t} \circ \Psi_{s, t}=\varphi_{s, t} \circ W_{s}, \quad 0 \leq s \leq t<+\infty,
$$

where $W_{\tau}(\zeta):=\exp (\zeta \log r(\tau))$ for all $\tau \geq 0$ and all $\zeta \in \mathbb{S}$.

In Section 5 we discuss the relationship between evolution families and Carathéodory ODEs. In particular, we prove (see Definitions 2.1 and 2.5)

Theorem 5.1. There is a one-to-one correspondence between the evolution families and semicomplete weak holomorphic vector fields. More precisely, every evolution family $\left(\left(D_{t}\right),\left(\varphi_{s, t}\right)\right)$ of order $d \in[1,+\infty]$ can be described as the non-autonomous semiflow of some semicomplete weak holomorphic vector field $G$ of order $d$ in $\mathcal{D}:=$ $\left\{(z, t): t \geq 0, z \in D_{t}\right\}$. Conversely, every such semiflow constitutes an evolution family.

Furthermore, in Theorem5.6 we establish, for the case when all the annuli $D_{t}$ are non-degenerate, a constructive characterization of semicomplete weak holomorphic vector fields in $\mathcal{D}$ analogous to the non-autonomous version of the Berkson-Porta

\footnotetext{
${ }^{1}$ The class of semicomplete weak holomorphic vector fields in $\mathbb{D}$ coincides with that of Herglotz vector fields. However, we prefer to avoid using the term "Herglotz vector field" in the doubly connected context.
} 
representation [10, Theorem 4.8], which characterizes Herglotz vector fields in the unit disk. Namely

Theorem 5.6. Let $d \in[1,+\infty]$, and let $\left(D_{t}\right)=\left(\mathbb{A}_{r(t)}\right)$ be a non-degenerate canonical domain system of order $d$. Then a function $G: \mathcal{D} \rightarrow \mathbb{C}$, where $\mathcal{D}:=$ $\left\{(z, t): t \geq 0, z \in D_{t}\right\}$, is a semicomplete weak holomorphic vector field of order $d$ if and only if there exist functions $p: \mathcal{D} \rightarrow \mathbb{C}$ and $C:[0,+\infty) \rightarrow \mathbb{R}$ such that:

(i) $G(w, t)=w\left[i C(t)+r^{\prime}(t) p(w, t) / r(t)\right]$ for a.e. $t \geq 0$ and all $w \in D_{t}$;

(ii) for each $w \in D:=\bigcup_{t \geq 0} D_{t}$ the function $p(w, \cdot)$ is measurable in $E_{w}:=$ $\{t \geq 0:(w, t) \in \mathcal{D}\}$

(iii) for each $t \geq 0$ the function $p(\cdot, t)$ belongs to the class $\mathcal{V}_{r(t)}$;

(iv) $C \in L_{\text {loc }}^{d}([0,+\infty), \mathbb{R})$.

As mentioned above, a part of our proofs rely on lifting evolution families from a system of annuli to a simply connected domain $D$. This technique, together with some new results on evolution families in $D$, is developed in Section 3 .

Moreover, we include an auxiliary Section 2 on Carathéodory differential equations driven by weak holomorphic vector fields. The first part contains standard facts about solutions to those ODEs that we regard as known. However, we include a sketch of the proofs, because to the best of our knowledge, no literature gives a direct proof of these results formulated exactly as we need. The second part is devoted to the case of the ODEs which are semicomplete to the right. It contains an exact characterization of the solutions, which is applied later to prove Theorem 5.1 .

In Section 6 we consider a number of examples. In particular, we prove that, in contrast to the simply connected case, an evolution family over a system of annuli can share any finite number of fixed points.

Finally, in Section 7 we briefly discuss how our results are related to the achievements of Komatu, Goluzin, Li En Pir, and Lebedev.

\section{Holomorphic Carathéodory differential equations}

In this section we obtain a characterization of solutions to Carathéodory ordinary differential equations driven by holomorphic vector fields.

Let $E \subset \mathbb{R}$ be a non-empty interval, bounded or unbounded, and consider a connected relatively open subset $\mathcal{D}$ of the set $\mathbb{C} \times E$. We fix $E$ and $\mathcal{D}$ throughout this section. In this paper we apply the results of this section for the case $E:=$ $[0,+\infty)$ and $\mathcal{D}:=\mathbb{D} \times[0,+\infty)$ or $\mathcal{D}:=\{(z, t): t \geq 0,0<|z|<r(t)\}$, where $r:[0,+\infty) \rightarrow[0,1)$ is non-increasing and continuous. However, we prefer to consider the general case, which might be useful for further studies and at the same time does not require any substantial modification of the argument.

By $A C^{d}(X, Y)$, where $X \subset \mathbb{R}, Y \subseteq \mathbb{C}$ and $d \in[1,+\infty]$, we denote the class of all locally absolutely continuous functions $f: X \rightarrow Y$ such that the derivative $f^{\prime}$ belongs to $L_{\text {loc }}^{d}(X)$. Further, denote by $D\left(z_{0}, r\right)$, where $r>0$ and $z_{0} \in \mathbb{C}$, the disk $\left\{z:\left|z-z_{0}\right|<r\right\}$, and let $\mathbb{D}:=D(0,1)$ and $\mathbb{T}:=\partial \mathbb{D}$.

2.1. Weak holomorphic vector fields and Carathéodory ODEs. Carathéodory's theory of ODEs is a well-established area of analysis, see, e.g., [15, §II.1], 19, $\S I .1],[30$, Ch. 18], or [39, §VIII.8]. The facts we state below can be considered well known. However, these results do not seem to be proved earlier in the literature 
directly and explicitly in the form that our context requires. That is why we prefer to sketch the proofs.

We start by introducing the class of vector fields we deal with.

Definition 2.1. Let $d \in[1,+\infty]$. A function $G: \mathcal{D} \rightarrow \mathbb{C}$ is said to be a weak holomorphic vector field of order $d$ in the domain $\mathcal{D}$, if it satisfies the following conditions:

WHVF1. For each $z \in \mathbb{C}$ the function $G(z, \cdot)$ is measurable in $E_{z}:=\{t:(z, t) \in$ $\mathcal{D}\}$.

WHVF2. For each $t \in E$ the function $G(\cdot, t)$ is holomorphic in $D_{t}:=\{z:(z, t) \in$ $\mathcal{D}\}$.

WHVF3. For each compact set $K \subset \mathcal{D}$ there exists a non-negative function $k_{K} \in L^{d}\left(\operatorname{pr}_{\mathbb{R}}(K), \mathbb{R}\right)$, where $\operatorname{pr}_{\mathbb{R}}(K):=\{t \in E: \exists z \in \mathbb{C} \quad(z, t) \in K\}$, such that

$|G(z, t)| \leq k_{K}(t), \quad$ for almost every $t \in \operatorname{pr}_{\mathbb{R}}(K)$ and $(z, t) \in K$.

Remark 2.2. It is easy to see that condition WHVF3 in Definition 2.1 is equivalent to

WHVF3' For any closed disk $B \subset \mathbb{C}$ and any compact interval $I \subset E$ such that $B \times I \subset \mathcal{D}$ there exists a non-negative function $k_{B, I} \in L^{d}(I, \mathbb{R})$ such that $|G(z, t)| \leq k_{B, I}(t)$ for almost every $t \in I$ and all $z \in B$.

Given a weak holomorphic vector field $G$ in $\mathcal{D}$ and a point $(z, s) \in \mathcal{D}$, let us consider the following initial value problem for a Carathéodory ODE

$$
\dot{w}(t)=G(w(t), t), \quad w(s)=z .
$$

Problem (2.1) is equivalent (see, e.g., [19, §I.1]) to the integral equation $\mathcal{L}_{z}^{s} w=w$, where

$$
\left(\mathcal{L}_{z}^{s} w\right)(t):=z+\int_{s}^{t} G(w(\xi), \xi) d \xi
$$

is well defined for any continuous function $w: J \rightarrow \mathbb{C}$ such that $J \subset E$ is an interval, $s \in J$, and $(w(t), t) \in \mathcal{D}$ for all $t \in J$.

Theorem 2.3. Let $G$ be a weak holomorphic vector field in $\mathcal{D}$ of order $d \in[1,+\infty]$. Then the following statements hold:

(i) For any $(z, s) \in \mathcal{D}$, the initial value problem (2.1) has a unique maximal solution $t \mapsto w_{s}^{*}(z, t)$. In other words, there exists an interval $J_{*}(z, s) \subset$ $E, s \in J_{*}(z, s)$, and a solution $w_{s}^{*}(z, \cdot)$ to the problem (2.1) defined in $J_{*}(z, s)$ such that any other solution $t \mapsto w_{0}(t)$ to (2.1) is a restriction of $w_{s}^{*}(z, \cdot)$.

(ii) For any $(z, s) \in \mathcal{D}$ the maximal solution $w_{s}^{*}(z, \cdot)$ belongs to $A C^{d}\left(J_{*}(z, s), \mathbb{C}\right)$.

(iii) For any $(z, s) \in \mathcal{D}$ such that $\sup J_{*}(z, s)<\sup E$ there exists no compact set $K \subset \mathcal{D}$ such that $\left\{\left(w_{s}^{*}(z, t), t\right): t \in J_{*}(z, s), t \geq s\right\} \subset K$.

(iv) For any $\left(z_{0}, s_{0}\right) \in \mathcal{D}$ and any $t_{0} \in J_{*}\left(z_{0}, s_{0}\right)$ there exists $\varepsilon>0$ such that $t_{0} \in J_{*}\left(z, s_{0}\right)$ whenever $\left|z-z_{0}\right|<\varepsilon$. Moreover, the function $z \mapsto$ $w_{s_{0}}^{*}\left(z, t_{0}\right)$ is holomorphic and injective in $D\left(z_{0}, \varepsilon\right)$. 
(v) For any $\left(z_{0}, s_{0}\right) \in \mathcal{D}$ and any $t_{0} \in J_{*}\left(z_{0}, s_{0}\right)$ there exists $\epsilon>0$ such that the mapping $(z, s, t) \mapsto w_{s}^{*}(z, t)$ is well defined for all $(z, s, t) \in U(\epsilon):=$ $\left\{(z, s, t): z \in D\left(z_{0}, \epsilon\right), s, t \in E,\left|t-t_{0}\right|<\epsilon,\left|s-s_{0}\right|<\epsilon\right\}$. Moreover, this mapping is continuous at the point $\left(z_{0}, s_{0}, t_{0}\right)$.

First we prove the following lemma.

Lemma 2.4. Let $G: \mathcal{D} \rightarrow \mathbb{C}$ be a weak holomorphic vector field of order $d \in$ $[1,+\infty]$, and let $K \subset \mathcal{D}$ be a compact set. Suppose $I \subset E$ is a compact interval and $B:=\overline{D\left(z_{0}, 2 R\right)}$ is a closed disk such that $B \times I \subset K$. Then for any $s \in I$ the following assertions hold:

(a) for each $z \in D\left(z_{0}, R\right)$ and each sufficiently small interval $J \subset I, J \ni s$, there exists a unique solution $J \ni t \mapsto w_{s}(z, t)$ to the initial value problem (2.1);

(b) for each $z \in D\left(z_{0}, R\right)$ the solution to (2.1) can be continued all over the interval $J_{h}(s):=(s-h, s+h) \cap I$, where $h=h(G, K, R)>0$ is a constant independent of $I, s$, and $z$;

(c) for each $t \in J_{h}$ the function $w_{s}(\cdot, t)$ is holomorphic in $D\left(z_{0}, R\right)$.

Moreover,

(d) the mapping $(z, s, t) \mapsto w_{s}(z, t)$ is continuous on $A:=\{(z, s, t): z \in$ $\left.D\left(z_{0}, R\right), s, t \in I, \quad|s-t|<h\right\}$.

Proof. First of all let us note that, since $K \subset \mathcal{D}$ is compact, there exists $\delta>0$ such that

$$
\bigcup_{(z, t) \in K}(\overline{D(z, \delta)} \times\{t\}) \Subset \mathcal{D} .
$$

Then the argument of the proof of [10, Lemma 4.2] can be easily adapted to show that there exists a non-negative function $\hat{k}_{K} \in L^{d}\left(\operatorname{pr}_{\mathbb{R}}(K), \mathbb{R}\right)$ depending only on $G$ and $K$ such that

$$
\left|G\left(z_{2}, t\right)-G\left(z_{1}, t\right)\right| \leq \hat{k}_{K}(t)\left|z_{2}-z_{1}\right| \quad \text { whenever }\left(z_{1}, t\right),\left(z_{2}, t\right) \in K .
$$

Choose any $\alpha \in(0,1)$. There exists $h>0$ that fulfills the following two conditions:

$$
\begin{aligned}
& \left|\int_{s}^{t} k_{K}(\xi) d \xi\right|<R \quad \text { whenever } s, t \in I \text { and }|t-s|<h, \\
& \left|\int_{s}^{t} \hat{k}_{K}(\xi) d \xi\right| \leq \alpha \quad \text { whenever } s, t \in I \text { and }|t-s|<h .
\end{aligned}
$$

By $C(X, Y), X$ and $Y$ topological spaces, let us denote the class of all continuous functions from $X$ to $Y$. Fix any $s \in I$. Given an interval $J \subset J_{h}(s)$ with $s \in J$, from (2.3) -(2.5) it easily follows that for any $z \in D\left(z_{0}, R\right)$, the operator $\mathcal{L}_{z}^{s}$, given by (2.2), is a contracting self-mapping of $C(J, B)$ endowed with the Chebyshev metric $\rho\left(w_{2}, w_{1}\right):=\sup _{t \in J}\left|w_{2}(t)-w_{1}(t)\right|$. The metric space $M:=(C(J, B), \rho)$ is complete. Hence the Banach fixed point theorem implies statements (a) and (b) of Lemma 2.4.

The proof of $(\mathrm{d})$ is similar. The operator $\mathcal{L}$, defined by the formula $(\mathcal{L} w)(z, t ; s)$ $:=\left(\mathcal{L}_{z}^{s} w(z, \cdot ; s)\right)(t)$, is a contractive self-mapping of $C(A, B)$ endowed with the metric $\tilde{\rho}\left(w_{2}, w_{1}\right):=\sup _{(z, s, t) \in A}\left|w_{2}(z, t ; s)-w_{1}(z, t ; s)\right|$. The metric space $\tilde{M}=$ $(C(A, B), \tilde{\rho})$ is complete. Hence it contains a solution to the equation $\mathcal{L} w=w$, 
which, by virtue of (a), has to coincide with $A \ni(z, s, t) \mapsto w_{s}(z, t)$. This proves (d).

We are left with statement (c). Clearly, it is sufficient to show that $\mathcal{L}$ maps into itself the closed subspace $\tilde{M}_{\text {hol }}$ of $\tilde{M}$ consisting of all maps from $\tilde{M}$ which are holomorphic in $z$.

So consider any $w \in \tilde{M}_{\text {hol }}$. Fix an arbitrary $(z, s, t) \in A$. For all $\xi \in J_{h}(s)$ and all $\omega \in U:=D\left(0,\left(R-\left|z-z_{0}\right|\right) / 2\right) \backslash\{0\}$ we have

$$
\left|\frac{G(w(z+\omega, \xi ; s), \xi)-G(w(z, \xi ; s), \xi)}{\omega}\right| \leq C \hat{k}_{K}(\xi)
$$

with some constant $C=C(z)>0$ not depending on $\omega$ and $\xi$. Here we combined (2.3) with the fact that the family $\left\{w(\cdot, \xi ; s): \xi \in J_{h}(s)\right\}$ is normal in $D\left(z_{0}, R\right)$ and hence the mapping $(\omega, \xi) \mapsto(w(z+\omega, \xi ; s)-w(z, \xi ; s)) / \omega$ is bounded on $U \times J_{h}(s)$.

By construction,

$$
\begin{aligned}
& \frac{(\mathcal{L} w)(z+\omega, t ; s)-(\mathcal{L} w)(z, t ; s)}{\omega} \\
&=1+\int_{s}^{t} \frac{G(w(z+\omega, \xi ; s), \xi)-G(w(z, \xi ; s), \xi)}{\omega} d \xi .
\end{aligned}
$$

From (2.6) it follows that we can apply Lebesgue's dominated convergence theorem to conclude that there exists a finite limit of the integral in (2.7) as $\omega \rightarrow 0$. Thus $(\mathcal{L} w)(\cdot, t ; s)$ is differentiable in the complex sense in $D\left(z_{0}, R\right)$. This completes the proof.

Proof of Theorem 2.3. Assertion (a) of Lemma 2.4 implies that for any $(z, s) \in \mathcal{D}$ there exists a unique local solution to (2.1). Thus statement (i) of Theorem 2.3 follows in the same way as in the classical theory of ODEs.

Now fix any $(z, s) \in \mathcal{D}$ and take an arbitrary compact interval $I \subset J_{*}(z, s)$. By condition WHVF3 with $K:=\left\{\left(w_{s}^{*}(z, \xi), \xi\right): \xi \in I\right\}$, for any $t_{1}, t_{2} \in I$ such that $t_{1}<t_{2}$, we have

$$
\left|w_{s}^{*}\left(z, t_{2}\right)-w_{s}^{*}\left(z, t_{1}\right)\right| \leq \int_{t_{1}}^{t_{2}}\left|G\left(w_{s}^{*}(z, \xi), \xi\right)\right| d \xi \leq \int_{t_{1}}^{t_{2}} k_{K}(\xi) d \xi,
$$

where $k_{K} \in L^{d}(I, \mathbb{R})$ and does not depend on $t_{1}$ and $t_{2}$. This proves statement (ii).

Let $K \subset \mathcal{D}$ be any compact set. Then there is another compact set $K_{1} \subset \mathcal{D}$ and constants $R>0$ and $\delta>0$ such that $\overline{D(z, 2 R)} \times([s-\delta, s+\delta] \cap E) \subset K_{1}$ whenever $(z, s) \in K$. We claim that any solution $J \ni t \mapsto w_{0}(t)$ to the equation $\dot{w}=G(w, t)$ such that $\left(w_{0}(t), t\right) \in K$ for all $t \in J$, is extendable to a neighborhood of $t^{*}:=\sup J$ provided $t^{*}<\sup E$. Indeed, by assertion (b) of Lemma 2.4 applied with $K_{1}$ substituted for $K$, there exists $\delta_{1} \in(0, \delta]$ such that for any $s \in J$ the initial value problem (2.1) with $z:=w_{0}(s)$ has a solution defined on the interval $\left(s-\delta_{1}, s+\delta_{1}\right) \cap E$. In view of the uniqueness of solution to (2.1), this proves our claim, which, in its turn, implies statement (iii) of the theorem.

Let us prove statement (iv). For simplicity we may assume that $t_{0}>s_{0}$. Let $J:=\left[s_{0}, t_{0}\right]$. Taking $w_{0}:=\left.w_{s_{0}}^{*}\left(z_{0}, \cdot\right)\right|_{J}$ and $K:=\left\{\left(w_{0}(t), t\right): t \in J\right\}$ in the above argument and using assertion (c) of Lemma 2.4 we can conclude that there exists a finite increasing sequence $\left(s_{j}\right)_{j=0}^{n}$ starting with $s_{0}$ and finishing with $s_{n}:=t_{0}$ such 
that for each $j=1, \ldots, n$ the function $w_{s_{j-1}}^{*}(z, t)$ is well defined and holomorphic in $z$ for all $t \in I_{j}:=\left[s_{j-1}, s_{j}\right]$ and all $z \in D\left(w_{0}\left(s_{j-1}\right), R\right)$. Since $w_{s_{j-1}}^{*}\left(w_{0}\left(s_{j-1}\right), s_{j}\right)=$ $w_{0}\left(s_{j}\right)$, there exists $\varepsilon>0$ such that the composition $f(z):=w_{s_{n-1}}^{*}\left(\cdot, s_{n}\right) \circ \ldots \circ$ $w_{s_{1}}^{*}\left(\cdot, s_{2}\right) \circ w_{s_{0}}^{*}\left(\cdot, s_{1}\right)$ is well defined and holomorphic in $D\left(z_{0}, \varepsilon\right)$. It follows that $w_{s_{0}}^{*}\left(\cdot, t_{0}\right)$ is also well defined in $D\left(z_{0}, \varepsilon\right)$ and coincides there with $f$. The fact that $w_{s_{0}}^{*}\left(\cdot, t_{0}\right)$ is injective follows from the uniqueness of the solution in the same way as in the classical theory of ODEs.

We are left with the proof of statement (v). By statement (iv) the map $w_{s_{0}}^{*}\left(\cdot, t_{0}\right)$ is defined in $D\left(z_{0}, \varepsilon\right)$ and is continuous at the point $z_{0}$. It follows from assertions (b) and (d) of Lemma 2.4 that there exists $\varepsilon_{1}>0$ such that the map $(z, s) \mapsto w_{s}\left(z, s_{0}\right)$ is well defined in $\mathcal{O}_{\varepsilon_{1}}\left(z_{0}, s_{0}\right):=\left\{(z, s):\left|z-z_{0}\right|<\varepsilon_{1},\left|s-s_{0}\right|<\varepsilon_{1}, s \in E\right\}$ and continuous at $\left(z_{0}, s_{0}\right)$. Hence the $\operatorname{map}(z, s) \mapsto f(z, s):=w_{s_{0}}^{*}\left(w_{s}^{*}\left(z, s_{0}\right), t_{0}\right)$ is well defined in $\mathcal{O}_{\varepsilon_{2}}\left(z_{0}, s_{0}\right)$ for some $\varepsilon_{2}>0$ and continuous at the point $\left(z_{0}, s_{0}\right)$. Denote $\zeta_{0}:=w_{s_{0}}^{*}\left(z_{0}, t_{0}\right)$. Again by assertions (b) and (d) of Lemma 2.4 the map $(\zeta, t) \mapsto w_{t_{0}}^{*}(\zeta, t)$ is well defined in $\mathcal{O}_{\varepsilon_{3}}\left(\zeta_{0}, t_{0}\right)$ for some $\varepsilon_{3}>0$ and continuous at $\left(\zeta_{0}, t_{0}\right)$. Thus the map $(z, s, t) \mapsto g(z, s, t):=w_{t_{0}}^{*}(f(z, s), t)$ is well defined in $U(\epsilon)$ for some $\epsilon>0$ and continuous at the point $\left(z_{0}, s_{0}, t_{0}\right)$. Now to finish the proof, it remains to notice that $g$ is a restriction of the mapping $(z, s, t) \mapsto w_{s}^{*}(z, t)$.

\subsection{Semicomplete weak holomorphic vector fields and families of holo-} morphic functions generated by them. In this subsection we consider weak holomorphic vector fields $G$ for which the solution to the initial value problem (2.1) exists globally to the right. Our proofs take advantage of the methods used in [10, 11, 12, 14. Without loss of generality we adopt the following

Assumption. For any $t \in E$ the set $D_{t}:=\{z:(z, t) \in \mathcal{D}\}$ is not empty. For simplicity we will assume that all $D_{t}$ 's are domains, which is enough for our purpose. However, our arguments (with minimal changes) are also valid for the case when some of the $D_{t}$ 's are not necessarily connected.

Recall that by Theorem 2.3 , for any $(z, s) \in \mathcal{D}$ all solutions to the initial value problem (2.1) can be obtained as restrictions of the unique maximal solution $J_{*}(z, s) \ni t \mapsto w_{s}^{*}(z, t) \in \mathbb{C}$.

Definition 2.5. A weak holomorphic vector field $G: \mathcal{D} \rightarrow \mathbb{C}$ (of some order $d \in$ $[1,+\infty])$ is said to be semicomplete if for any $(z, s) \in \mathcal{D}$, the inclusion $J_{*}(z, s) \supset$ $E^{s}:=E \cap[s,+\infty)$ takes place, i.e., the initial value problem (2.1) has a solution defined everywhere in $E^{s}$.

In the following proposition we establish some important properties of the nonautonomous semiflows generated by semicomplete weak holomorphic vector fields.

Proposition 2.6. Let $G: \mathcal{D} \rightarrow \mathbb{C}$ be a semicomplete weak holomorphic vector field of order $d \in[1,+\infty]$. Then the formula $\varphi_{s, t}(z):=w_{s}^{*}(z, t)$ defines a family $\left(\varphi_{s, t}\right)_{s \in E, t \in E^{s}}$ of mappings $\varphi_{s, t}: D_{s} \rightarrow D_{t}$ such that the following assertions hold:

(i) $\varphi_{s, t}$ is holomorphic in $D_{s}$ for any $s \in E$ and any $t \in E^{s}$;

(ii) $\varphi_{s, s}=\mathrm{id}_{D_{s}}$ for any $s \in E$;

(iii) $\varphi_{s, t}=\varphi_{u, t} \circ \varphi_{s, u}$ for any $s, u, t \in E$ such that $s \leq u \leq t$; 
(iv) for any compact set $K \subset \mathcal{D}$ there exists a non-negative function $\tilde{k}_{K} \in$ $L_{\text {loc }}^{d}(E, \mathbb{R})$ such that

$$
\left|\varphi_{s, t}(z)-\varphi_{s, u}(z)\right| \leq \int_{u}^{t} \tilde{k}_{K}(\xi) d \xi
$$

for any $u, t \in E$ and any $(z, s) \in K$ satisfying $s \leq u \leq t$.

Proof. The proof of the first three assertions is straightforward from previous results. Indeed, assertion (i) follows from Theorem 2.3(iv); assertion (ii) holds by the very definition of $w_{s}^{*}(z, t)$; and (iii) is a consequence of Theorem [2.3)(i).

We are left with assertion (iv). Let $K \subset \mathcal{D}$ be a compact set. For each $T \in E^{s}$ the set $\widetilde{K}(T):=\left\{\left(\varphi_{s, t}(z), t\right):(z, s) \in K, s \leq t \leq T\right\} \subset \mathcal{D}$ is also compact, since $(z, s, t) \mapsto \varphi_{s, t}(z)$ is continuous by Theorem $2.3(\mathrm{v})$.

Denote $s_{0}:=\min \operatorname{pr}_{\mathbb{R}}(K)$. Then, by condition WHVF3 of Definition 2.1, there exists a non-negative function $k_{\widetilde{K}(T)} \in L^{d}\left(\left[s_{0}, T\right], \mathbb{R}\right)$ such that $|G(w, t)| \leq k_{\widetilde{K}(T)}(t)$ for any $(w, t) \in \widetilde{K}(T)$. Extend $k_{\widetilde{K}(T)}$ to $E$ by setting $k_{\widetilde{K}(T)}(t)=0$ for all $t \in$ $E \backslash\left[s_{0}, T\right]$. Take any non-decreasing sequence $\left(T_{n}\right) \subset E$ such that $T_{1}>s_{0}$ and $T_{n} \rightarrow$ $\sup E$ as $n \rightarrow+\infty$. Define $\tilde{k}_{K}:=\chi_{\left[s_{0}, T_{1}\right]} k_{\widetilde{K}\left(T_{1}\right)}+\sum_{n=2}^{+\infty} \chi_{\left(T_{n-1}, T_{n}\right]} k_{\widetilde{K}\left(T_{n}\right)}$, where $\chi_{A}$ stands for the characteristic function of a set $A$. Obviously, $\tilde{k}_{K} \in L_{\text {loc }}^{d}(E, \mathbb{R})$ and $\left|G\left(\varphi_{s, t}(z), t\right)\right| \leq \tilde{k}_{K}(t)$ for all $(z, s) \in K$ and all $t \in E^{s}$.

To complete the proof it remains to recall that

$$
\varphi_{s, t}(z)-\varphi_{s, u}(z)=\int_{u}^{t} G\left(\varphi_{s, \xi}(z), \xi\right) d \xi
$$

for any $(z, s) \in \mathcal{D}$ and any $u, t \in E^{s}$.

The converse of Proposition 2.6 is also true: the properties (i)-(iv) turn out to be characteristic. The exact formulation of this fact uses the following two notions from the analysis of infinitely dimensional vector-functions of a real variable.

Let $U$ and $W$ be some domains in the complex plane $\mathbb{C}$ and $I \subset \mathbb{R}$ an interval containing at least two different points. $\mathrm{By} \operatorname{Hol}(U, W)$ we denote the set of all holomorphic functions from $U$ to $W$ endowed with the topology of the uniform convergence on compacta.

Definition 2.7. A mapping $\varphi: I \rightarrow \operatorname{Hol}(U, W)$ will be called absolutely continuous on $I$ if for any $\varepsilon>0$ and any compact set $K \subset U$ there exists $\delta=\delta(\varepsilon, K)>0$ such that for any finite set of pairwise disjoint intervals $\left(I_{j}\right)_{j=1}^{n}, I_{j}:=\left(a_{j}, b_{j}\right), a_{j}, b_{j} \in I$, we have

$$
\sum_{j=1}^{n}\left(b_{j}-a_{j}\right)<\delta \Longrightarrow \sum_{j=1}^{n}\left\|\varphi\left(b_{j}\right)-\varphi\left(a_{j}\right)\right\|_{K}<\varepsilon,
$$

where $\|\cdot\|_{K}$ stands for the Chebyshev norm on $K$, i.e., $\|f\|_{K}:=\sup _{z \in K}|f(z)|$ for any bounded function $f: K \rightarrow \mathbb{C}$.

A mapping $\varphi: I \rightarrow \operatorname{Hol}(U, W)$ will be called locally absolutely continuous if $\varphi$ is absolutely continuous on each compact interval $J \subset I$.

Definition 2.8. A mapping $\varphi: I \rightarrow \operatorname{Hol}(U, W)$ will be called differentiable at a point $t_{0} \in I$ if there exists a function $g \in \operatorname{Hol}(U, \mathbb{C})$ such that

$$
\left(\varphi(t)-\varphi\left(t_{0}\right)\right) /\left(t-t_{0}\right) \rightarrow g
$$


in the topology of $\operatorname{Hol}(U, \mathbb{C})$, i.e.,

$$
\left\|g-\left(\varphi(t)-\varphi\left(t_{0}\right)\right) /\left(t-t_{0}\right)\right\|_{K} \rightarrow 0 \quad \text { for any } K \Subset U,
$$

as $t \rightarrow t_{0}, t \in I \backslash\left\{t_{0}\right\}$. The function $g$ is the derivative of $\varphi$ at the point $t_{0}$ and will be denoted by $(d \varphi / d t)\left(t_{0}\right)$ or $\dot{\varphi}\left(t_{0}\right)$.

Remark 2.9. Let $G: U_{1} \rightarrow U$ and $F: W \rightarrow W_{1}$ be two arbitrary holomorphic functions. Define the mapping $C_{F, G}: \operatorname{Hol}(U, W) \rightarrow \operatorname{Hol}\left(U_{1}, W_{1}\right)$ by setting $C_{F, G}(g):=F \circ g \circ G$ for all $g \in \operatorname{Hol}(U, W)$. It is easy to show that $C_{F, G} \circ \varphi$ is locally absolutely continuous provided so is $\varphi: I \rightarrow \mathrm{Hol}(U, W)$. The same is true for the notion of differentiability: if $\varphi: I \rightarrow \operatorname{Hol}(U, W)$ is differentiable at some point $t_{0} \in E$, then $C_{F, G} \circ \varphi$ is also differentiable at $t_{0}$ and $\left(d\left(C_{F, G} \circ \varphi\right) / d t\right)\left(t_{0}\right)=$ $\left(F^{\prime} \circ \varphi\left(t_{0}\right) \circ G\right)\left((d \varphi / d t)\left(t_{0}\right) \circ G\right)$.

Now we can state the following

Theorem 2.10. Let $d \in[1,+\infty]$, and let $\left(\varphi_{s, t}\right)_{s \in E, t \in E^{s}}$ be a family of maps $\varphi_{s, t}$ : $D_{s} \rightarrow D_{t}$ such that assertions (i)-(iv) of Proposition 2.6 hold. Then there exist a null-set $N \subset E$ and a semicomplete weak holomorphic vector field $G: \mathcal{D} \rightarrow \mathbb{C}$ of order $d$ such that the following statements are true:

(a) for any $s \in E$ the map $E^{s} \ni t \mapsto \varphi_{s, t} \in \operatorname{Hol}\left(D_{s}, \mathbb{C}\right)$ is locally absolutely continuous;

(b) for any $s \in E^{\prime}:=E \backslash\{\sup E\}$ the map $E^{s} \ni t \mapsto \varphi_{s, t} \in \operatorname{Hol}\left(D_{s}, \mathbb{C}\right)$ is differentiable in $E^{s} \backslash N$;

(c) $d \varphi_{s, t} / d t=G(\cdot, t) \circ \varphi_{s, t}$ for all $s \in E^{\prime}$ and all $t \in E^{s} \backslash N$.

The proof of Theorem 2.10 is preceded by the following three lemmas.

Lemma 2.11. Under the conditions of Theorem 2.10, for each $s \in E$ and each $t \in E^{s}$, the function $\varphi_{s, t}$ is not constant in $D_{s}$.

Proof. Assume to the contrary that $\varphi_{s_{0}, t_{0}}$ is constant for some $s_{0}, t_{0} \in E$ with $s_{0} \leq t_{0}$. Take $u_{0}:=\left(s_{0}+t_{0}\right) / 2$. Then $\varphi_{s_{0}, t_{0}}=\varphi_{u_{0}, t_{0}} \circ \varphi_{s_{0}, u_{0}}$ by (iii). Hence either $\varphi_{s_{0}, u_{0}}$ is constant in $D_{s_{0}}$ or $\varphi_{u_{0}, t_{0}}$ is constant in $D_{u_{0}}$. Repeating this procedure several times if necessary, we see that one could assume from the very beginning that there exist $R>0$ and $z_{0} \in \mathbb{C}$ such that $K:=\overline{D\left(z_{0}, R\right)} \times\left[s_{0}, t_{0}\right] \subset \mathcal{D}$.

Combining (ii) and (iv), we see that there exists non-negative $\tilde{k}_{K} \in L_{\text {loc }}^{d}(E, \mathbb{R})$ such that $\left|\varphi_{s, t}(z)-z\right| \leq \int_{s}^{t} \tilde{k}_{K}(\xi) d \xi$ whenever $\left|z-z_{0}\right| \leq R$ and $s_{0} \leq s \leq t \leq t_{0}$. Again using bisections of the interval, we conclude that there exist $s, t \in\left[s_{0}, t_{0}\right]$ such that $s \leq t, \varphi_{s, t} \equiv$ const, and $\int_{s}^{t} \tilde{k}_{K}(\xi) d \xi<R$. Then $\left|\varphi_{s, t}(z)-z\right|<R$ for all $z \in \overline{D\left(z_{0}, R\right)}$, which implies that $\varphi_{s, t}\left(z_{0}+R\right) \neq \varphi_{s, t}\left(z_{0}-R\right)$. The contradiction finishes the proof.

Lemma 2.12. Let $z_{0} \in \mathbb{C}, t_{0} \in E$, and $E_{z_{0}} \cap\left(-\infty, t_{0}\right] \neq \emptyset$. Then under the conditions of Theorem 2.10 , the map $E_{z_{0}} \cap\left(-\infty, t_{0}\right] \ni s \mapsto \varphi_{s, t_{0}}\left(z_{0}\right)$ is continuous.

Proof. Fix an arbitrary $\left(z_{0}, s_{0}\right) \in \mathcal{D}$ and $t_{0} \in E^{s_{0}}$. We have to prove that $\varphi_{s, t_{0}}\left(z_{0}\right) \rightarrow \varphi_{s_{0}, t_{0}}\left(z_{0}\right)$ as $s \rightarrow s_{0}$. The proof is in two steps.

Step 1. First we prove continuity from the left. So assume that $s<s_{0}$ and that $s \in E_{z_{0}}$. From (ii) and (iv) it follows that $\varphi_{s, s_{0}}\left(z_{0}\right) \rightarrow z_{0}$ as $s \rightarrow s_{0}-0$. Hence by (iii), $\varphi_{s, t_{0}}\left(z_{0}\right)=\varphi_{s_{0}, t_{0}}\left(\varphi_{s, s_{0}}\left(z_{0}\right)\right) \rightarrow \varphi_{s_{0}, t_{0}}\left(z_{0}\right)$ as $s \rightarrow s_{0}-0$. 
Step 2. If $E \ni \sup E$ and $s_{0}=\sup E$, then the proof is finished. So we may suppose that $s_{0}<s<\sup E$. Choose any $s_{1}>s_{0}$ and $R>0$ such that $K:=$ $\overline{D\left(z_{0}, R\right)} \times\left[s_{0}, s_{1}\right] \subset \mathcal{D}$. Then it follows from (ii) and (iv) that $\varphi_{s_{0}, s}(z) \rightarrow z$ uniformly in $\overline{D\left(z_{0}, R\right)}$ as $s \rightarrow s_{0}+0, s<s_{1}$. Using Rouche's theorem, one can easily show that for all $s \in\left(s_{0}, s_{1}\right)$ close enough to $s_{0}$ there exists a function $\psi_{s_{0}, s}: D\left(z_{0}, R / 2\right) \rightarrow \mathbb{C}$ such that $\varphi_{s_{0}, s} \circ \psi_{s_{0}, s}=\mathrm{id}_{D\left(z_{0}, R / 2\right)}$ and that $\psi_{s_{0}, s}\left(z_{0}\right) \rightarrow z_{0}$ as $s \rightarrow s_{0}+0$. Then by (iii), $\varphi_{s, t_{0}}\left(z_{0}\right)=\varphi_{s_{0}, t_{0}}\left(\psi_{s_{0}, s}\left(z_{0}\right)\right) \rightarrow \varphi_{s_{0}, t_{0}}\left(z_{0}\right)$ as $s \rightarrow s_{0}+0$.

The proof is now finished.

Lemma 2.13. Let $h>0, z_{0} \in \mathbb{C}$, and $\tilde{E}_{z_{0}}:=E_{z_{0}} \cap \tilde{E} \neq \emptyset$, where $\tilde{E}:=\{s \in E$ : $s+h \in E\}$. Then under the conditions of Theorem 2.10, the map $\tilde{E}_{z_{0}} \ni s \mapsto$ $\varphi_{s, s+h}\left(z_{0}\right)$ is continuous.

Proof. Fix arbitrary $\left(z_{0}, s_{0}\right) \in \mathcal{D}$ with $s_{0} \in \tilde{E}$. Since $\mathcal{D}$ is relatively open in $\mathbb{C} \times E$, there exists $\delta \in(0, h / 2)$ such that $\left\{z_{0}\right\} \times I$, where $I:=\left[s_{0}-\delta, s_{0}+\delta\right] \cap \tilde{E}$, is a compact subset of $\mathcal{D}$. We have to prove that $\left|\varphi_{s, s+h}\left(z_{0}\right)-\varphi_{s_{0}, s_{0}+h}\left(z_{0}\right)\right| \rightarrow 0$ as $s \rightarrow s_{0}, s \in I$.

Note that $s_{0} \in I$. Moreover, for any $s \in I$, we have $s \leq s_{0}+h$. Hence by (iv) with $K:=\left\{z_{0}\right\} \times I$, we have

$$
\left|\varphi_{s, s+h}\left(z_{0}\right)-\varphi_{s, s_{0}+h}\left(z_{0}\right)\right| \leq\left|\int_{s_{0}+h}^{s+h} \tilde{k}_{K}(\xi) d \xi\right|
$$

where $\tilde{k}_{K} \in L_{\text {loc }}^{d}(E,[0,+\infty))$. Hence $\left|\varphi_{s, s+h}\left(z_{0}\right)-\varphi_{s, s_{0}+h}\left(z_{0}\right)\right| \rightarrow 0$ as $s \rightarrow s_{0}, s \in I$. To complete the proof it only remains to apply Lemma 2.12 with $t_{0}:=s_{0}+h$.

Proof of Theorem 2.10. First of all we notice that Theorem 2.10)(a) follows directly from Proposition 2.6(iv) applied with $K:=K^{\prime} \times\{s\}$, where $s \in E$ and $K^{\prime}$ is an arbitrary compact subset of $D_{s}$.

Let us prove Theorem 2.10(b). Consider any countable expanding system $\left(K_{n}\right)$ of compact sets $K_{n} \subset \mathcal{D}$ such that $\bigcup_{n \in \mathbb{N}}$ int $K_{n}=\mathcal{D}$, where int $A$ stands for the interior of a set $A$. (Such a system exists in any locally compact separable metric space.) To simplify the notation, we will denote by $k_{n}$ the function $\tilde{k}_{K_{n}}$ from Proposition 2.6(iv).

Fix any $t \in E$ and any $n \in \mathbb{N}$. If $k_{n}(t)<+\infty$ and $t$ is a Lebesgue point of $k_{n}$, then

$$
C_{n}(t):=\sup \left\{\left|\frac{1}{t^{\prime}-t} \int_{t^{\prime}}^{t} k_{n}(\xi) d \xi\right|: t^{\prime} \in E, 0<\left|t^{\prime}-t\right|<1\right\}<+\infty .
$$

Hence (2.8) holds for all $t \in E$ aside some null-set $M_{n} \subset E$ containing all nonLebesgue points of $k_{n}$. Set $M:=\bigcup_{n \in \mathbb{N}} M_{n}$.

Fix any $s \in E^{\prime}:=E \backslash\{\sup E\}$. Let $K \subset D_{s}$ be a compact set. Then, by construction, there exists $n \in \mathbb{N}$ such that $K \times\{s\} \subset K_{n}$. Therefore, by (2.8),

$$
\left\|\frac{\varphi_{s, t^{\prime}}-\varphi_{s, t}}{t^{\prime}-t}\right\|_{K} \leq\left|\frac{1}{t^{\prime}-t} \int_{t^{\prime}}^{t} k_{n}(\xi) d \xi\right| \leq C_{n}(t)<+\infty
$$

for all $t \in E^{s} \backslash M$ and all $t^{\prime} \in E^{s}$ such that $0<\left|t^{\prime}-t\right|<1$.

It follows from (a) that $E^{s} \ni t \mapsto \varphi_{s, t}(z)$ is locally absolutely continuous for any $(z, s) \in \mathcal{D}$. Hence there exists a null-set $N(z, s) \subset E^{s}$ such that $d \varphi_{s, t}(z) / d t$ exists for all $t \in E^{s} \backslash N(z, s)$. Fix an arbitrary $s \in E^{\prime}$ and take any sequence $\left(z_{k}\right) \subset D_{s}$ 
with at least one accumulation point in $D_{s}$. Set $N(s):=\left[\bigcup_{k \in \mathbb{N}} N\left(z_{k}, s\right)\right] \cup M$. Then, owing to (2.9), Vitali's principle implies that for any $t \in E^{s} \backslash N(s)$, there

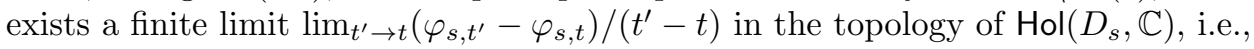
$t \mapsto \varphi_{s, t} \in \operatorname{Hol}\left(D_{s}, \mathbb{C}\right)$ is differentiable aside $N(s)$.

Now take any $s_{1} \leq s, s_{1} \in E$. We claim that $t \mapsto \varphi_{s, t} \in \operatorname{Hol}\left(D_{s}, \mathbb{C}\right)$ is also differentiable for all $t \in E^{s} \backslash N\left(s_{1}\right)$. Indeed, by the above argument, $t \mapsto \varphi_{s_{1}, t}$ is differentiable for all $t \in E^{s_{1}} \backslash N\left(s_{1}\right) \supset E^{s} \backslash N\left(s_{1}\right)$. Since $\varphi_{s_{1}, t}=\varphi_{s, t} \circ \varphi_{s_{1}, s}$ for all $t \in E^{s}$, we can conclude that $\left.t \mapsto \varphi_{s, t}\right|_{U}$, where $U:=\varphi_{s_{1}, s}\left(D_{s_{1}}\right)$, is differentiable for all $t \in E^{s} \backslash N\left(s_{1}\right)$. By Lemma 2.11, $U \subset D_{s}$ is a domain. Hence again using (2.9) and Vitali's principle, we conclude that $t \mapsto \varphi_{s, t}$ is differentiable in $t \in E \backslash N\left(s_{1}\right)$.

If $E \ni \inf E$, we set $s_{1}:=\min E$ and $N^{\prime}:=N\left(s_{1}\right)$. If $E \not \supset \inf E$, we take any decreasing sequence $\left(s_{n}\right) \subset E$ such that $s_{n} \rightarrow \inf E$ as $n \rightarrow+\infty$ and define $N^{\prime}:=\bigcup_{n \in \mathbb{N}} N\left(s_{n}\right)$.

Finally, for the case $E \not \supset \sup E$ we set $N:=N^{\prime}$. Otherwise, we set $N:=$ $N^{\prime} \cup\{\max E\}$. Now the proof of (b) is finished.

We are left with statement Theorem 2.10(c). Define the function $G: \mathcal{D} \rightarrow \mathbb{C}$ in the following way: $G(\cdot, t):=d \varphi_{s, t} /\left.d t\right|_{s:=t}$ for all $t \in E \backslash N$, and $G(\cdot, t) \equiv 0$ for all $t \in N$. Then, from assertion (iii), it immediately follows $d \varphi_{s, t} / d t=G(\cdot, t) \circ \varphi_{s, t}$ for all $t \in E \backslash N$ and all $s \in E \cap(-\infty, t]$. So it remains to show that $G$ is a semicomplete weak holomorphic field of order $d$.

First of all, $G(\cdot, t) \in \operatorname{Hol}\left(D_{t}, \mathbb{C}\right)$ for all $t \in E$ by construction. Further, for any fixed $z \in \mathbb{C}$ such that $E_{z} \neq \emptyset$, we have $n\left(\varphi_{s, s+1 / n}(z)-z\right) \rightarrow G(z, s)$ as $n \rightarrow+\infty$ for a.e. $s \in E_{z}$. By Lemma 2.13, for each fixed $n \in \mathbb{N}$ the function $s \mapsto \varphi_{s, s+1 / n}$ is continuous. It follows that $G(z, \cdot)$ is measurable on $E_{z}$. Therefore, $G$ satisfies conditions WHVF1 and WHVF2 from Definition 2.1.

Let us check condition WHVF3. Fix any compact set $K \subset \mathcal{D}$. By construction, there exists $n \in \mathbb{N}$ such that $K \subset K_{n}$. Let $(z, t) \in K$. If $t \in N$, then trivially we have $0=|G(z, t)| \leq k_{n}(t)$. So assume that $t \notin N$. By construction, $t$ is a Lebesgue point for $k_{n}$. Hence, on the one hand,

$$
Q\left(t, t^{\prime}\right):=\left|\frac{1}{t^{\prime}-t} \int_{t}^{t^{\prime}} k_{n}(\xi) d \xi\right| \rightarrow k_{n}(t) \quad \text { as } t^{\prime} \rightarrow t .
$$

On the other hand, $G(z, t)=\lim _{t^{\prime} \rightarrow t+0}\left(\varphi_{t, t^{\prime}}(z)-z\right) /\left(t^{\prime}-t\right)$. Passing in the inequality $\left|\varphi_{t, t^{\prime}}(z)-z\right| /\left|t^{\prime}-t\right| \leq Q\left(t, t^{\prime}\right)$ to the limit as $t^{\prime} \rightarrow t+0$, we again obtain $|G(z, t)| \leq k_{n}(t)$.

Note that $I(K):=\operatorname{pr}_{\mathbb{R}}(K)$ is compact. Thus to finish the proof of WHVF3, it is now sufficient to set $k_{K}:=\left.k_{n}\right|_{I(K)}$.

Finally, by the above arguments, for any $(z, s) \in \mathcal{D}, E^{s} \ni t \mapsto \varphi_{s, t}(z)$ solves the initial value problem (2.1). Thus the vector field $G$ is semicomplete. This finishes the proof.

\section{Evolution FAMILIES IN SIMPLY CONNECTED DOMAINS}

The notion of an evolution family is one of the three central notions in modern Loewner theory in simply connected domains. In this paper we will use the following definition of an evolution family in a domain of the complex plane, which for the case of the unit disk $\mathbb{D}$ was formulated in [10].

Definition 3.1. Let $D$ be a domain in $\mathbb{C}$ and $d \in[1,+\infty]$. A family $\left(\varphi_{s, t}\right)_{0 \leq s \leq t<+\infty}$ of holomorphic self-maps $\varphi_{s, t}: D \rightarrow D$ is said to be an evolution family of order $d$ 
(or, in short, $L^{d}$-evolution family) in the domain $D$ if it satisfies the following three conditions:

EF1. $\varphi_{s, s}=\mathrm{id}_{D}$;

EF2. $\varphi_{s, t}=\varphi_{u, t} \circ \varphi_{s, u}$ whenever $0 \leq s \leq u \leq t<+\infty$;

EF3. for any $T>0$ and any $z \in D$ there exists a non-negative function $k_{z, T} \in L^{d}([0, T], \mathbb{R})$ such that

$$
\left|\varphi_{s, t}(z)-\varphi_{s, u}(z)\right| \leq \int_{u}^{t} k_{z, T}(\xi) d \xi
$$

whenever $0 \leq s \leq u \leq t \leq T$.

One can show that all non-trivial cases of evolution families in a (fixed) domain can be reduced in one way or another to the case when $D=\mathbb{D}$ (see Proposition 5.15). We will not go into detail, except for stating the following result, which will be applied further in the paper. Its proof is rather standard and we omit it.

Proposition 3.2. Let $d \in[1,+\infty]$. Let $\left(\varphi_{s, t}\right)_{0 \leq s \leq t}$ be a family of holomorphic self-maps of the unit disk $\mathbb{D}$ and $F: \mathbb{D} \rightarrow \mathbb{C}$ any holomorphic univalent function. Then the formula $\Phi_{s, t}=F \circ \varphi_{s, t} \circ F^{-1}, t \geq 0, s \in[0, t]$, defines an $L^{d}$-evolution family in $D:=F(\mathbb{D})$ if and only if $\left(\varphi_{s, t}\right)$ is an $L^{d}$-evolution family in $\mathbb{D}$.

The authors of [10] established a deep relationship between evolution families in the unit disk $\mathbb{D}$ and Carathéodory non-autonomous differential equations, driven by the so-called Herglotz vector fields.

Definition 3.3. A Herglotz vector field of order $d$ (in short an $L^{d}$-Herglotz vector field) in a simply connected domain $D \varsubsetneqq \mathbb{C}$ is a weak holomorphic vector field $G$ of order $d$ in $\mathcal{D}:=D \times[0,+\infty)$ such that for a. e. fixed $t \geq 0$ the function $G(\cdot, t)$ is an infinitesimal generator in $D$.

Remark 3.4. For the notion of an infinitesimal generator and the theory behind it we refer the reader to [1, §1.4.1] or [10, Section 2]. According to the Berkson-Porta representation formula [9], the set of all infinitesimal generators in $\mathbb{D}$ coincides with the set of all functions given by the formula $G(z)=(\tau-z)(1-\bar{\tau} z) p(z)$, $z \in \mathbb{D}$, where $\tau$ is an arbitrary point in the closed unit disk $\overline{\mathbb{D}}$ and $p: \mathbb{D} \rightarrow$ $\mathbb{C}$ is an arbitrary holomorphic function satisfying the inequality $\operatorname{Re} p(z) \geq 0$ for all $z \in \mathbb{D}$. Moreover, if $F: \mathbb{D} \rightarrow \mathbb{C}$ is a holomorphic univalent function, then the infinitesimal generators in $D:=F(\mathbb{D})$ are exactly the functions $G_{D}$ given by the formula $G_{D}(w)=F^{\prime}\left(F^{-1}(w)\right) G\left(F^{-1}(w)\right), w \in D$, where $G$ is an arbitrary infinitesimal generator in $\mathbb{D}$. The non-autonomous version of this statement follows easily: given $d \in[1,+\infty]$, the function $G: \mathbb{D} \times[0,+\infty)$ is a Herglotz vector field of order $d$ in $\mathbb{D}$ if and only if the formula $G_{D}(w, t):=F^{\prime}\left(F^{-1}(w)\right) G\left(F^{-1}(w), t\right)$, $w \in D, t \geq 0$, defines a Herglotz vector field $G_{D}$ of order $d$ in the domain $D$.

Remark 3.5. One of the immediate consequences of the above remark is that an infinitesimal generator $G_{D}: D \rightarrow \mathbb{C}$ can have at most one zero in $D$ unless it vanishes identically.

In 10, it was proved that every Herglotz vector field in $\mathbb{D}$ is semicomplete [10, Theorem 4.4]. Moreover, there is a one-to-one correspondence between these vector fields and evolution families. Namely, for every Herglotz vector field $G$ : 
$\mathbb{D} \times[0,+\infty) \rightarrow \mathbb{C}$ of order $d$ there exists a unique $L^{d}$-evolution family $\left(\varphi_{s, t}\right)$ generated by the vector field $G$ in the following sense: for any $z \in \mathbb{D}$ and any $s \geq 0$, the function $[s,+\infty) \ni t \mapsto \varphi_{s, t}(z)$ solves the initial value problem $\dot{w}=G(w, t)$, $w(s)=z$ [10, Theorem 5.2]. Conversely, every $L^{d}$-evolution family $\left(\varphi_{s, t}\right)$ in $\mathbb{D}$ is generated by a unique (up to a null-set) Herglotz vector field $G: \mathbb{D} \times[0,+\infty) \rightarrow \mathbb{C}$ of order $d$ [10, Theorem 6.2]. Using Proposition 3.2 and Remark 3.4 one can easily conclude that these results also hold with $\mathbb{D}$ replaced by any simply connected domain $D \varsubsetneqq \mathbb{C}$. Hence, taking into account Proposition 2.6 with $\mathbb{D} \times[0,+\infty)$ substituted for $\mathcal{D}$, we can state Theorem 6.2 from [10] in the following, a bit stronger form:

Theorem 3.6. Let $\left(\varphi_{s, t}\right)$ be an $L^{d}$-evolution family in a simply connected domain $D \varsubsetneqq \mathbb{C}$. The following statements hold:

(i) For any $s \geq 0$, the mapping $[s,+\infty) \ni t \mapsto \varphi_{s, t} \in \operatorname{Hol}(D, D)$ is locally absolutely continuous.

(ii) Moreover, the above assertion (i) holds locally uniformly w.r.t. s, i.e., for any $T>0$ and any $K \Subset D$ there exists a non-negative function $k_{K, T} \in L^{d}([0, T], \mathbb{R})$, not depending on $s$, such that

$$
\left\|\varphi_{s, t}-\varphi_{s, u}\right\|_{K} \leq \int_{u}^{t} k_{K, T}(\xi) d \xi
$$

for any $s, u, t \in[0, T]$ such that $s \leq u \leq t$.

(iii) There exists a Herglotz vector field $G:[0,+\infty) \rightarrow \operatorname{Hol}(D, \mathbb{C}) ; t \mapsto G_{t}$ of order $d$ and a null-set $N \subset[0,+\infty)$ such that for any $s \geq 0$ the mapping $[s,+\infty) \ni t \mapsto \varphi_{s, t} \in \operatorname{Hol}(D, \mathbb{C})$ is differentiable aside $N$ and for each $t \in[s,+\infty) \backslash N$ we have $(d / d t) \varphi_{s, t}=G_{t} \circ \varphi_{s, t}$.

We finish this section with the proof of a modification of [16, Proposition 2.10], which does not seem to appear in the literature earlier.

Proposition 3.7. Let $d \in[1,+\infty], D \varsubsetneqq \mathbb{C}$ be a simply connected domain and $\left(\Phi_{s, t}\right)_{0 \leq s \leq t<+\infty} \subset \operatorname{Hol}(D, D)$. Suppose that $\left(\Phi_{s, t}\right)$ satisfies conditions EF1 and EF2 from Definition 3.1. Let $\zeta_{1}, \zeta_{2} \in D$ and $\zeta_{1} \neq \zeta_{2}$. If the functions $t \mapsto \Phi_{0, t}\left(\zeta_{1}\right)$ and $t \mapsto \Phi_{0, t}\left(\zeta_{2}\right)$ belong to $A C^{d}([0,+\infty), D)$ and $\Phi_{0, t}\left(\zeta_{1}\right) \neq \Phi_{0, t}\left(\zeta_{2}\right)$ for all $t \geq 0$, then $\left(\Phi_{s, t}\right)$ is an evolution family of order $d$ in the domain $D$.

To prove the above proposition we need the following

Lemma 3.8. There exists a universal constant $C>0$ such that for any holomorphic map $\varphi: \mathbb{D} \rightarrow \mathbb{D}$ with $\varphi(0)=0$, any $r \in(0,1)$ and any $\zeta_{0} \in \mathbb{D} \backslash\{0\}$, the following inequality holds:

$$
|\varphi(\zeta)-\zeta| \leq \frac{C}{\left|\zeta_{0}\right|\left(1-\left|\zeta_{0}\right|^{2}\right)\left(1-r^{2}\right)}\left|\varphi\left(\zeta_{0}\right)-\zeta_{0}\right|, \quad|\zeta| \leq r
$$

Proof. We fix arbitrary $\zeta_{0} \in \mathbb{D} \backslash\{0\}, r \in(0,1)$, and $\varphi \in \operatorname{Hol}(\mathbb{D}, \mathbb{D})$ with $\varphi(0)=0$. If $\left|\varphi\left(\zeta_{0}\right)-\zeta_{0}\right| \geq\left|\zeta_{0}\right|$, then (3.1) holds trivially with any $C \geq 2$.

So we can assume that $\left|\varphi\left(\zeta_{0}\right)-\zeta_{0}\right|<\left|\zeta_{0}\right|$. Then $\left|\varphi\left(\zeta_{0}\right)+\zeta_{0}\right|>\left|\zeta_{0}\right|>0$. Hence, it follows from the Schwarz lemma that $\varphi^{\prime}(0) \neq-1$ and that $\varphi(\zeta) / \zeta \in \overline{\mathbb{D}} \backslash\{-1\}$ for all $\zeta \in \mathbb{D} \backslash\{0\}$.

Therefore, the function $p(\zeta):=(1-\varphi(\zeta) / \zeta) /(1+\varphi(\zeta) / \zeta)$, extended to the origin by continuity, is holomorphic in $\mathbb{D}$ and there satisfies the inequality $\operatorname{Re} p(\zeta) \geq 0$. 
Hence, [38, ineq. (11) on p. 40] implies that for any $|\zeta| \leq r$,

$$
\begin{array}{r}
|p(\zeta)| \leq\left|\operatorname{Im} p\left(\zeta_{0}\right)\right|+\left|\operatorname{Re} p\left(\zeta_{0}\right)\right| \frac{1+\left|\frac{\zeta_{0}-\zeta}{1-\overline{\zeta_{0}} \zeta}\right|}{1-\left|\frac{\zeta_{0}-\zeta}{1-\overline{\zeta_{0}} \zeta}\right|} \leq \sqrt{2}\left|p\left(\zeta_{0}\right)\right| \frac{1+\left|\frac{\zeta_{0}-\zeta}{1-\overline{\zeta_{0}} \zeta}\right|}{1-\left|\frac{\zeta_{0}-\zeta}{1-\overline{\zeta_{0}} \zeta}\right|} \\
=\sqrt{2}\left|p\left(\zeta_{0}\right)\right| \frac{\left(\left|1-\overline{\zeta_{0}} \zeta\right|+\left|\zeta_{0}-\zeta\right|\right)^{2}}{\left(1-\left|\zeta_{0}\right|^{2}\right)\left(1-|\zeta|^{2}\right)} \leq\left|p\left(\zeta_{0}\right)\right| \frac{16 \sqrt{2}}{\left(1-\left|\zeta_{0}\right|^{2}\right)\left(1-r^{2}\right)} .
\end{array}
$$

Finally, bearing in mind that $\left|\varphi\left(\zeta_{0}\right)+\zeta_{0}\right|>\left|\zeta_{0}\right|$ we deduce that, whenever $0<|\zeta| \leq$ $r$,

$$
\begin{aligned}
|\varphi(\zeta)-\zeta| & =|\zeta+\varphi(\zeta)||p(\zeta)| \leq \frac{32 \sqrt{2}}{\left(1-\left|\zeta_{0}\right|^{2}\right)\left(1-r^{2}\right)} \frac{\left|\zeta_{0}-\varphi\left(\zeta_{0}\right)\right|}{\left|\zeta_{0}+\varphi\left(\zeta_{0}\right)\right|} \\
& \leq \frac{32 \sqrt{2}}{\left|\zeta_{0}\right|\left(1-\left|\zeta_{0}\right|^{2}\right)\left(1-r^{2}\right)}\left|\varphi\left(\zeta_{0}\right)-\zeta_{0}\right| .
\end{aligned}
$$

This finishes the proof.

Proof of Proposition 3.7. First we prove the proposition for the case $D:=\mathbb{D}$. For the sake of convenience we change the notation used in Proposition 3.7 So let $\left(\varphi_{s, t}\right)_{0 \leq s \leq t<+\infty} \subset \operatorname{Hol}(\mathbb{D}, \mathbb{D}), z_{1}, z_{2} \in \mathbb{D}, d \in[1,+\infty]$. Assume that $(1) \varphi_{s, s}=\mathrm{id}_{\mathbb{D}}$ for all $s \geq 0$; (2) $\varphi_{s, t}=\varphi_{u, t} \circ \varphi_{s, u}$ whenever $0 \leq s \leq u \leq t<+\infty$; (3) $t \mapsto \varphi_{0, t}\left(z_{1}\right)$ and $t \mapsto \varphi_{0, t}\left(z_{2}\right)$ belong to $A C^{d}([0,+\infty), \mathbb{D}) ;(4) \varphi_{0, t}\left(z_{1}\right) \neq \varphi_{0, t}\left(z_{2}\right)$ for all $t \geq 0$. We have to prove that $\left(\varphi_{s, t}\right)$ is an $L^{d}$-evolution family in $\mathbb{D}$.

Write

$$
h_{t}(z):=\frac{z+a(t)}{1+\overline{a(t)} z}, \quad a(t):=\varphi_{0, t}\left(z_{1}\right) .
$$

Define $\psi_{s, t}:=h_{t}^{-1} \circ \varphi_{s, t} \circ h_{s}$. We claim that $\left(\psi_{s, t}\right)$ is an $L^{d}$-evolution family. Clearly, $\left(\psi_{s, t}\right)$ satisfies conditions EF1 and EF2 from Definition 3.1. Moreover, $\psi_{s, t}(0)=0$ for any $s \geq 0$ and any $t \geq s$. To check condition EF3, we note that $t \mapsto \zeta_{0}(t):=\psi_{0, t}\left(z_{0}\right) \in \mathbb{D}$, where $z_{0}:=h_{0}^{-1}\left(z_{2}\right)$, belongs to $A C^{d}([0,+\infty), \mathbb{D})$ and does not vanish.

Now take arbitrary $T>0$. Fix any $z \in \mathbb{D}$ and any $s, u, t \in[0, T]$ such that $s \leq u \leq t$. Denote $r:=|z|$ and $\varepsilon:=\min _{u \in[0, T]}\left|\zeta_{0}(u)\right|>0$. Applying Lemma 3.8 with $\varphi:=\psi_{u, t}, \zeta:=\psi_{s, u}(z), \zeta_{0}:=\zeta_{0}(u)$ and taking into account that $|\zeta| \leq|z|=r$ and $\left|\zeta_{0}(u)\right| \leq\left|z_{0}\right|$ by the Schawrz lemma, we get

$$
\begin{aligned}
\left|\psi_{s, t}(z)-\psi_{s, u}(z)\right|=\left|\psi_{u, t}(\zeta)-\zeta\right| & \leq \frac{C}{\left|\zeta_{0}(u)\right|\left(1-\left|\zeta_{0}(u)\right|^{2}\right)\left(1-r^{2}\right)}\left|\psi_{u, t}\left(\zeta_{0}\right)-\zeta_{0}\right| \\
& \leq \frac{C}{\varepsilon\left(1-\left|z_{0}\right|^{2}\right)\left(1-r^{2}\right)}\left|\psi_{0, t}\left(z_{0}\right)-\psi_{0, u}\left(z_{0}\right)\right| .
\end{aligned}
$$

This shows that $\left(\psi_{s, t}\right)$ satisfies EF3 and hence it is an $L^{d}$-evolution family. Now we apply [16. Lemma 2.8] to conclude that $\left(\varphi_{s, t}\right)$ is also an evolution family of order $d$.

The proof for the case $D:=\mathbb{D}$ is complete. For arbitrary simply connected domains $D \varsubsetneqq \mathbb{C}$, the proposition now follows from the Riemann Mapping Theorem and Proposition 3.2 


\section{Evolution FAMILIES OVER SYSTEMS OF DOUBLY CONNECTED DOMAINS}

4.1. Definition of an evolution family in the doubly connected case. As we mentioned in the introduction, the most important new property of Loewner evolution in the multiply connected case is that the canonical domain has to evolve in time, while in the simply connected case the conformal type does not change. On the level of evolution families one can explain this phenomenon by the fact that all the families satisfying Definition 3.1 for $D:=\mathbb{A}_{r}$ with some fixed $r \in(0,1)$ are exhausted by rotations (see Example6.1). So, instead of one fixed reference domain, we consider families of reference domains. A natural choice of doubly connected reference domains are the annuli $\mathbb{A}_{r}$, where $r \in[0,1$ ). (Note we do not exclude the case $r=0$.) With each annulus $\mathbb{A}_{r}$ we can associate a one-generated torsion-free Fuchsian group $\Gamma$ such that $\mathbb{A}_{r}$ is conformally equivalent to $\mathbb{D} / \Gamma$. This group is unique up to conjugation by a Möbius transformation, and the conjugation classes are uniquely defined by the multiplier $\lambda$ of the generator of $\Gamma$. It is not difficult to calculate that $\lambda=e^{-2 \pi \omega(r)}$, where

$$
\omega(r):= \begin{cases}-\pi / \log r, & \text { if } r \in(0,1) \\ 0, & \text { if } r=0\end{cases}
$$

Hence it is natural to consider families of annuli $\left(\mathbb{A}_{r(t)}\right)_{t \geq 0}$ assuming some regularity of the function $t \mapsto \omega(r(t))$. Namely, we introduce the following

Definition 4.1. Let $d \in[1,+\infty]$ and $\left(D_{t}\right)_{t \geq 0}$ be a family of annuli $D_{t}:=\mathbb{A}_{r(t)}$. We will say that $\left(D_{t}\right)$ is a (doubly connected) canonical domain system of order $d$ (or in short, a canonical $L^{d}$-system) if the function $t \mapsto \omega(r(t))$ belongs to $A C^{d}([0,+\infty),[0,+\infty))$ and does not increase. If $r(t) \equiv 0$, then the canonical domain system $\left(D_{t}\right)$ will be called degenerate. If on the contrary $r(t)$ does not vanish, then $\left(D_{t}\right)$ will be called non-degenerate. Finally, if there exists $T>0$ such that $r(t)>0$ for all $t \in[0, T)$ and $r(t)=0$ for all $t \geq T$, then we will say that $\left(D_{t}\right)$ is of mixed type.

Remark 4.2. The condition that $t \mapsto \omega(r(t))$ is of class $A C^{d}$ implies that $t \mapsto$ $r(t)$ also belongs to $A C^{d}([0,+\infty),[0,1))$. In the non-degenerate case, i.e., when $r(t)>0$ for all $t \geq 0$, or if $d=1$, then the converse is also true and we can replace $\omega(r(t))$ by $r(t)$ in the above definition. However, in general this is not the case and for some auxiliary statements (e.g., for Lemma 4.10) the condition $\omega \circ r \in A C^{d}([0,+\infty),[0,+\infty))$ is essential even if we assume that $t \mapsto r(t)$ is of class $A C^{d}$. At the same time we do not know whether any of our main results would fail to hold in the mixed case with $d>1$ if in Definition 4.1 one places a weaker condition $r \in A C^{d}([0,+\infty),[0,1))$ instead of $\omega \circ r \in A C^{d}([0,+\infty),[0,+\infty))$.

Now we can introduce the definition of an evolution family for the doubly connected setting.

Definition 4.3. Let $\left(D_{t}\right)_{t \geq 0}$ be a canonical domain system of order $d \in[1,+\infty]$. A family $\left(\varphi_{s, t}\right)_{0 \leq s \leq t<+\infty}$ of holomorphic mappings $\varphi_{s, t}: D_{s} \rightarrow D_{t}$ is said to be an evolution family of order $d$ over $\left(D_{t}\right)$ (in short, an $L^{d}$-evolution family) if the following conditions are satisfied:

EF1. $\varphi_{s, s}=\mathrm{id}_{D_{s}}$;

EF2. $\varphi_{s, t}=\varphi_{u, t} \circ \varphi_{s, u}$ whenever $0 \leq s \leq u \leq t<+\infty$; 
EF3. for any closed interval $I:=[S, T] \subset[0,+\infty)$ and any $z \in D_{S}$ there exists a non-negative function $k_{z, I} \in L^{d}([S, T], \mathbb{R})$ such that

$$
\left|\varphi_{s, u}(z)-\varphi_{s, t}(z)\right| \leq \int_{u}^{t} k_{z, I}(\xi) d \xi
$$

whenever $S \leq s \leq u \leq t \leq T$.

Suppressing the language we will also refer to the pair $\mathcal{E}:=\left(\left(D_{t}\right),\left(\varphi_{s, t}\right)\right)$ as an evolution family of order $d$ and apply terms degenerate, non-degenerate, of mixed type to $\mathcal{E}$ whenever they are applicable to the canonical domain system $\left(D_{t}\right)$.

The notion of an evolution family over a degenerate canonical domain system given by Definition 4.3 is the same as the notion of an evolution family in the domain $D:=\mathbb{D}^{*}$ given by Definition 3.1. It is not difficult to see that this case is equivalent to that of evolution families in the unit disk fixing the origin. We will discuss it briefly in Subsection 5.2, while the main attention in this paper will be paid to the non-degenerate case.

4.2. Lifting evolution families to a simply connected domain. Given a canonical domain system $\left(D_{t}\right)$ and a family $\left(\varphi_{s, t}\right)$ satisfying algebraic conditions EF1 and EF2 from Definition 4.3, there is a lifting of $\left(\varphi_{s, t}\right)$ to the upper half-plane $\mathbb{H}:=\{z: \operatorname{Im} z>0\}$ (or any other hyperbolic simply connected domain) satisfying conditions EF1 and EF2 from Definition 3.1. Under some additional conditions such lifting is unique. Technical details can be seen in the next subsection. An important role in our arguments is played by the class $\mathbb{M}\left(r_{1}, r_{2}\right)$ of all functions $\psi \in \operatorname{Hol}\left(\mathbb{A}_{r_{1}}, \mathbb{A}_{r_{2}}\right), 1>r_{1} \geq r_{2} \geq 0$, such that $I(\psi \circ \gamma)=I(\gamma)$ for any oriented closed curve $\gamma \subset \mathbb{A}_{r_{1}}$, where $I(\gamma)$ stands for the index of the point $z=0$ w.r.t. $\gamma$.

The lifting technique allows us to apply the theory of evolution families in simply connected domains to establish some useful results in the doubly connected case. One of these results is the following analogue of Proposition 3.7. which gives a sufficient (and in fact necessary) condition for $\left(\varphi_{s, t}\right)$ to be an $L^{d}$-evolution family over $\left(D_{t}\right)$.

Theorem 4.4. Let $\left(D_{t}\right)=\left(\mathbb{A}_{r(t)}\right)$ be a canonical domain system of order $d \in$ $[1,+\infty]$, and let $\left(\varphi_{s, t}\right)_{0 \leq s \leq t}$ be a family of holomorphic functions $\varphi_{s, t}: D_{s} \rightarrow D_{t}$ satisfying conditions EF1 and EF2 in Definition 4.3. Suppose that at least one of the following conditions holds:

(a) For each $t>0$, each $s_{0} \in[0, t)$, and any $z \in D_{s_{0}}$, the mapping $\left[s_{0}, t\right] \ni$ $s \mapsto \varphi_{s, t}(z) \in \mathbb{D}^{*}$ is continuous.

(b) For each $s \geq 0$ and any $z \in D_{s}$, the mapping $[s,+\infty) \ni t \mapsto \varphi_{s, t}(z) \in$ $\mathbb{D}^{*}$ is continuous.

(c) For each $s \geq 0$ and $t \geq s$, the function $\varphi_{s, t}$ belongs to the class $\mathbb{M}(r(s), r(t))$.

If there exists a point $z_{0} \in D_{0}$ such that the function $[0,+\infty) \ni t \mapsto \varphi_{0, t}\left(z_{0}\right) \in \mathbb{D}^{*}$ belongs to $A C^{d}\left([0,+\infty), \mathbb{D}^{*}\right)$, then $\left(\varphi_{s, t}\right)$ is an $L^{d}$-evolution family over $\left(D_{t}\right)$.

Using the lifting technique, we can also extend assertion (ii) of Theorem 3.6 to the doubly connected setting.

Proposition 4.5. Let $\left(\varphi_{s, t}\right)$ be an $L^{d}$-evolution family over a canonical $L^{d}$-system $\left(D_{t}\right)=\left(\mathbb{A}_{r(t)}\right)$. Then for any closed interval $I:=[S, T] \subset[0,+\infty)$ and any compact 
set $K \subset D_{S}$ there exists a non-negative function $k_{K, I} \in L^{d}(I, \mathbb{R})$ such that

$$
\left\|\varphi_{s, t}-\varphi_{s, u}\right\|_{K} \leq \int_{u}^{t} k_{K, I}(\xi) d \xi
$$

for all $s, u, t \in I$ satisfying $s \leq u \leq t$.

Our study of the vector fields corresponding to non-degenerate $L^{d}$-evolution families is also based on the lifting technique. A suitable geometry of the covering space for non-degenerate case is the one of the $\operatorname{strip} \mathbb{S}:=\{z: 0<\operatorname{Re} z<1\}$. This is a motivation for following

Theorem 4.6. Let $\left(D_{t}\right)=\left(\mathbb{A}_{r(t)}\right)$ be a non-degenerate canonical domain system of order $d \in[1,+\infty]$. Then for any $L^{d}$-evolution family $\left(\varphi_{s, t}\right)$ over $\left(D_{t}\right)$ there exists a unique $L^{d}$-evolution family $\left(\Psi_{s, t}\right)$ in the strip $\mathbb{S}$ such that

$$
W_{t} \circ \Psi_{s, t}=\varphi_{s, t} \circ W_{s}, \quad 0 \leq s \leq t<+\infty,
$$

where $W_{\tau}(\zeta):=\exp (\zeta \log r(\tau))$ for all $\tau \geq 0$ and all $\zeta \in \mathbb{S}$.

The proofs are given in Subsection 4.4 and based on four lemmas we are going to establish in the next subsection.

4.3. Some auxiliary statements. The following lemma was proved for the case $D:=\mathbb{D}$ in [10, Proposition 3.5]. The same argument with obvious modifications works for any hyperbolic domain $D$. Therefore we omit the proof.

Lemma 4.7. Let $\left(\Phi_{s, t}\right)$ be an $L^{d}$-evolution family in a hyperbolic domain $D$ (i.e., admitting a hyperbolic metric). Then the mapping $\{(s, t): 0 \leq s \leq t \leq T\} \ni$ $(s, t) \mapsto \Phi_{s, t} \in \operatorname{Hol}(D, D)$ is continuous.

Lemma 4.8. Suppose $\left(\left(D_{t}\right),\left(\varphi_{s, t}\right)\right)$ is an evolution family of order $d \in[1,+\infty]$. Let $s \geq 0$. Then the following statements are true:

(i) for each $z \in D_{s}$ the function $t \mapsto \varphi_{s, t}(z)$ belongs to $A C^{d}([s,+\infty), \mathbb{C})$;

(ii) the mapping $t \mapsto \varphi_{s, t} \in \operatorname{Hol}\left(\mathbb{A}_{r(s)}, \mathbb{D}^{*}\right), \mathbb{D}^{*}:=\mathbb{D} \backslash\{0\}$, is continuous in $[s,+\infty)$;

(iii) $\varphi_{s, t} \in \mathbb{M}(r(s), r(t))$ for any $t \geq s$;

(iv) $\varphi_{s, t}$ is univalent in $D_{s}$ for any $t \geq s$.

Proof. To prove (i) we need only to apply condition EF3 from Definition 4.3 for $S:=s$. Fix $t \geq s$. From (i) it now follows that $\varphi_{s, u}(z) \rightarrow \varphi_{s, t}(z)$ pointwise in $D_{s}$ as $u \rightarrow t$. The functions $\varphi_{s, t}, t \geq s$, form (for fixed $s \geq 0$ ) a normal family in $D_{s}$. Therefore, the pointwise convergence implies convergence of $\varphi_{s, u}$ to $\varphi_{s, t}$ in $\operatorname{Hol}\left(D_{s}, \mathbb{C}\right)$. This proves (ii).

Now let us take any closed curve $\gamma:[0,1] \rightarrow D_{s}$. Fix $t \geq s$. Recall that $\varphi_{s, s}=\mathrm{id}_{D_{s}}$. Hence, (ii) implies that $g(u, x):=\varphi_{s, u}(\gamma(x)), u \in[s, t], x \in[0,1]$, is a homotopical deformation of the curve $\gamma$ into the curve $\varphi_{s, t} \circ \gamma$ within the domain $D_{t}$. It follows that $I\left(\varphi_{s, t} \circ \gamma\right)=I(\gamma)$. Since $\gamma$ is chosen arbitrarily, (iii) is now also proved.

To prove (iv) we argue as in [11, Proposition 3]. Assume that there exist $s \geq$ $0, t>s$, and $z_{1}, z_{2} \in D_{s}$ such that $z_{1} \neq z_{2}$ but $\varphi_{s, t}\left(z_{1}\right)=\varphi_{s, t}\left(z_{2}\right)$. Denote $\zeta_{j}(u):=\varphi_{s, u}\left(z_{j}\right), j=1,2$. Let $u_{0}:=\inf \left\{u \in[s, t]: \zeta_{1}(u)=\zeta_{2}(u)\right\}$. Clearly, 
$u_{0} \in[s, t]$. From (i) we know that the functions $\zeta_{j}$ are continuous. Therefore, $\zeta_{1}\left(u_{0}\right)=\zeta_{2}\left(u_{0}\right):=\zeta_{0}$. In particular, $u_{0} \neq s$. At the same time, by construction

$$
\zeta_{1}(u) \neq \zeta_{2}(u), \quad u \in\left[s, u_{0}\right) .
$$

Let $U \ni \zeta_{0}$ be any domain such that $\bar{U} \subset D_{u_{0}}$. Then there exists $u_{1} \in\left[s, u_{0}\right)$ such that $\left\{\zeta_{1}(u), \zeta_{2}(u)\right\} \subset U \subset D_{u}$ for all $u \in\left[u_{1}, u_{0}\right]$. In particular, the functions $\varphi_{u, u_{0}}$ are well defined and holomorphic in $U$ for all $u \in\left[u_{1}, u_{0}\right]$. By condition EF2 in Definition 4.3 ,

$$
\varphi_{u, u_{0}}\left(\zeta_{1}(u)\right)=\varphi_{u, u_{0}}\left(\zeta_{2}(u)\right)=\zeta_{0}, \quad u \in\left[u_{1}, u_{0}\right] .
$$

Now we claim that $\left.\varphi_{u, u_{0}}\right|_{U} \rightarrow \mathrm{id}_{U}$ in $\operatorname{Hol}(U, \mathbb{C})$ as $u \rightarrow u_{0}-0$. The pointwise convergence $\varphi_{u, u_{0}}(z) \rightarrow z, z \in U$, is a consequence of condition EF3 in Definition 4.3 applied with $\left(s, u, u, u_{0}, t\right)$ substituted for $(S, s, u, t, T)$. Since the functions $\varphi_{u, u_{0}}$, $u \in\left[u_{1}, u_{0}\right]$, form a normal family in $U$, the pointwise convergence implies convergence in $\operatorname{Hol}(U, \mathbb{C})$. In particular, it follows that given a sufficiently small open neighborhood $W$ of the point $\zeta_{0}$, the function $\varphi_{u, u_{0}}$ is univalent on $W$ provided $u$ is sufficiently close to $u_{0}$. The fact that this statement contradicts relations (4.3) and (4.4) proves assertion (iv).

Lemma 4.9. If either condition (a) or (b) in Theorem 4.4holds, then condition (c) in Theorem 4.4 holds as well.

Proof. Assume first that (b) holds. Fix any $s \geq 0$. By normality of the family $\left(\varphi_{s, t}\right)_{t \geq 0}$ in $D_{s}$, assertion (b) implies that the map $[s,+\infty) \ni t \mapsto \varphi_{s, t} \in \operatorname{Hol}\left(D_{s}, \mathbb{D}^{*}\right)$ is continuous. Hence as in the proof of Lemma 4.8 we can conclude that for any fixed $t \geq s$ the map $[0,1] \times D_{s} \ni(\theta, z) \mapsto \varphi_{s, t(\theta)}(z) \in \mathbb{D}^{*}$, where $t(\theta):=(1-\theta) t+\theta s$, is a homotopical family in $\mathbb{D}^{*}$ joining $\varphi_{s, t}$ with $\mathrm{id}_{D_{s}}$. It follows immediately that $\varphi_{s, t} \in \mathbb{M}(r(s), r(t))$. This proves that $(\mathrm{b}) \Rightarrow(\mathrm{c})$.

The proof of the implication (a) $\Rightarrow(\mathrm{c})$ is similar. Combining (a) with the normality argument, we can conclude that for each $s \geq 0$ and $t \geq s$ the map $[0,1] \times D_{s} \ni$ $(\theta, z) \mapsto \varphi_{s(\theta), t}(z) \in \mathbb{D}^{*}$, where $s(\theta):=(1-\theta) s+\theta t$, is a homotopical family in $\mathbb{D}^{*}$ joining $\varphi_{s, t}$ with $\mathrm{id}_{D_{s}}$. Again (c) follows immediately.

Denote by $\mathbb{S}_{r}, r \in(0,1)$, the strip $\{z: \log r<\operatorname{Re} z<0\}$ and by $\mathbb{S}_{0}$ the left half-plane $\{z: \operatorname{Re} z<0\}$.

Lemma 4.10. Under the conditions of Theorem 4.4 there exists an evolution family $\left(\Phi_{s, t}\right)$ of order $d$ in $\mathbb{H}$ such that for all $s \geq 0$ and all $t \geq s$ we have

$$
B_{t} \circ \Phi_{s, t}=\varphi_{s, t} \circ B_{s},
$$

where $B_{\tau}(w):=\exp Q\left(w, \omega_{\tau}\right), \tau \geq 0$, and

$$
\begin{gathered}
Q(w, \omega):=\frac{i}{\omega} \log \frac{1+\omega w}{1-\omega w}, Q(w, 0):=2 i w, \\
\omega_{\tau}:=\omega(r(\tau))= \begin{cases}-\pi / \log r(\tau), & \text { if } r(\tau)>0, \\
0, & \text { if } r(\tau)=0 .\end{cases}
\end{gathered}
$$

Remark 4.11. By log in the formula for the function $Q$ in the above lemma we mean the single-valued branch of the logarithm in $\mathbb{C} \backslash(-\infty, 0]$ that vanishes at $\zeta=1$. Hence the function $Q$ being extended by continuity to $\omega=0$ is well defined and holomorphic in the domain $\left\{(w, \omega) \in \mathbb{C}^{2}: \omega w \notin(-\infty,-1] \cup[1,+\infty)\right\}$. For each $\omega \geq 0$ the function $Q(\cdot, \omega)$ maps $\mathbb{H}$ conformally onto the strip $\mathbb{S}_{r}$, where $r:=e^{-\pi / \omega}$, if $\omega>0$, or onto the left half-plane $\mathbb{S}_{0}$ if $\omega=0$. 
Proof of Lemma 4.10. Owing to Lemma 4.9, we can assume that condition (c) from Theorem 4.4 takes place. Let us first construct the family $\left(\Phi_{s, t}\right)$ and then prove that it is an $L^{d}$-evolution family.

Denote by $R(\cdot, \omega)$ the function inverse to $Q(\cdot, \omega)$, i.e.,

$$
R(\zeta, \omega)=\frac{1}{\omega} \frac{e^{-i \zeta \omega}-1}{e^{-i \zeta \omega}+1}
$$

for $\omega \neq 0$, and $R(\zeta, 0)=-i \zeta / 2$ when $\omega=0$. Therefore $R$ is holomorphic in $\mathbb{C}^{2} \backslash\{(\zeta, \omega): \zeta \omega=\pi(n+1 / 2)$ for some $n \in \mathbb{Z}\}$. Consider the curve $[0,+\infty) \ni t \mapsto$ $z(t):=\varphi_{0, t}\left(z_{0}\right) \in \mathbb{D}^{*}$, and let $t \mapsto \zeta(t)$ be any of its liftings w.r.t. the covering map exp $: \mathbb{S}_{0} \rightarrow \mathbb{D}^{*}$. Finally, define $w(t):=R\left(\zeta(t), \omega_{t}\right)$ for all $t \geq 0$, where $\omega_{t}$ is introduced in the statement of Lemma 4.10. Then $t \mapsto w(t) \in \mathbb{H}$ is continuous and $B_{t}(w(t))=z(t)$ for all $t \geq 0$.

Fix any $s \geq 0$ and any $t \geq s$. Taking into account EF2, we have $\left(\varphi_{s, t} \circ\right.$ $\left.B_{s}\right)(w(s))=z(t)$. Hence $\left(\varphi_{s, t} \circ B_{s}\right)(w(s))=B_{t}(w(t))$. Note that according to Remark 4.11, $B_{t}: \mathbb{H} \rightarrow \mathbb{A}_{r(t)}$ is a covering map. It follows that there exists a unique lifting $F$ of $\varphi_{s, t} \circ B_{s}: \mathbb{H} \rightarrow \mathbb{D}^{*}$ w.r.t. $B_{t}$ which takes $w(s)$ to $w(t)$. Now put $\Phi_{s, t}:=F$.

The above argument defines a family $\left(\Phi_{s, t}\right)_{0 \leq s \leq t} \subset \mathrm{Hol}(\mathbb{H}, \mathbb{H})$. Equality (4.5) takes place by construction, while conditions EF1 and EF2 for $\left(\Phi_{s, t}\right)$ follow from conditions EF1 and EF2 for $\varphi_{s, t}$ and the uniqueness of the lifting. Now according to Theorem 3.7 it remains for us to find two points $w_{1}, w_{2} \in \mathbb{H}$ such that the functions $t \mapsto w_{1}(t):=\Phi_{0, t}\left(w_{1}\right)$ and $t \mapsto w_{2}(t):=\Phi_{0, t}\left(w_{2}\right)$ are of class $A C^{d}$ and $w_{1}(t) \neq w_{2}(t)$ for all $t \geq 0$.

Put $w_{1}:=R\left(\zeta(0), \omega_{0}\right), w_{2}:=R\left(\zeta(0)+2 \pi i, \omega_{0}\right)$. Then by construction, $w_{1}(t)=$ $w(t)$ for all $t \geq 0$. We claim that

$$
w_{2}(t)=R(\zeta(t)+2 \pi i, \omega(t)) \text { for all } t \geq 0 .
$$

The above equality is obviously equivalent to stating that $\phi_{t}(\zeta(0)+2 \pi i)=\zeta(t)+2 \pi i$, where $\phi_{t}=Q\left(\cdot, \omega_{t}\right) \circ \Phi_{0, t} \circ R\left(\cdot, \omega_{0}\right)$. Note that $\phi_{t}(\zeta(0))=\zeta(t)$. Hence to prove (4.6) it is sufficient now to show that

$$
\phi_{t}(\zeta+2 \pi i)=\phi_{t}(\zeta)+2 \pi i \text { for all } \zeta \in \mathbb{S}_{r(0)} \text { and all } t \geq 0 .
$$

Recall that for each $s \geq 0$ and $t \geq s$, the function $\Phi_{s, t}$ was constructed to be a lifting of $\varphi_{s, t} \circ B_{s}: \mathbb{H} \rightarrow \mathbb{D}^{*}$ w.r.t. $B_{t}$. Therefore, for each $t \geq 0$, the function $\phi_{t}$ is the lifting of $\left.\varphi_{0, t} \circ \exp \right|_{\mathbb{S}_{r(0)}}$ w.r.t. $\left.\exp \right|_{\mathbb{S}_{r(t)}}$. It follows that for each fixed $\zeta \in \mathbb{S}_{r(0)}$ the curve $\phi_{t} \circ \gamma$, where $\gamma:[0,1] \rightarrow \mathbb{S}_{r(0)} ; \theta \mapsto \zeta+2 \pi i \theta$, is a lifting of the curve $\gamma_{1}:=\varphi_{0, t} \circ \exp \circ \gamma$ w.r.t. $\left.\exp \right|_{\mathbb{S}_{r(t)}}$. Consequently, taking into account that $\varphi_{0, t} \in \mathbb{M}(r(0), r(t))$, we get

$$
\phi_{t}(\zeta+2 \pi i)-\phi_{t}(\zeta)=\int_{\gamma_{1}} \frac{d z}{z}=2 \pi i I\left(\gamma_{1}\right)=2 \pi i I(\exp \circ \gamma)=2 \pi i,
$$

where $I(\cdot)$ stands for the index of the origin w.r.t. a curve.

Now it remains to show that $t \mapsto w_{1}(t)$ and $t \mapsto w_{2}(t)$ are of class $A C^{d}$. Recall that $t \mapsto \zeta(t)$ is a lifting of $t \mapsto \varphi_{0, t}\left(z_{0}\right)$, which is of class $A C^{d}$. Hence $t \mapsto \zeta(t)$ is of class $A C^{d}$ as well. Finally, by definition $t \mapsto \omega_{t}$ is also of class $A C^{d}$. Therefore $w_{1}(t)=w(t)=R\left(\zeta(t), \omega_{t}\right)$ is locally absolutely continuous and the derivative

$$
\frac{d w_{1}(t)}{d t}=R_{\zeta}^{\prime}\left(\zeta(t), \omega_{t}\right) \frac{d \zeta(t)}{d t}+R_{\omega}^{\prime}\left(\zeta(t), \omega_{t}\right) \frac{d \omega_{t}}{d t}
$$


belongs to $L_{\text {loc }}^{d}([0,+\infty), \mathbb{C})$, i.e., $w_{1} \in A C^{d}([0,+\infty), \mathbb{H})$. By a similar argument, $w_{2}(t) \in A C^{d}([0,+\infty), \mathbb{H})$. The proof is now complete.

\subsection{Proofs.}

Proof of Theorem 4.4. We have to show that $\left(\varphi_{s, t}\right)$ satisfies EF3. To this end we take advantage of Lemma 4.10 stating existence of an $L^{d}$-evolution family $\left(\Phi_{s, t}\right)$ in $\mathbb{H}$ such that (4.5) holds. Below we use the notation introduced in the statement and proof of this lemma.

Equality (4.5) can be written in the form

$$
\exp \circ \phi_{s, t}=\varphi_{s, t} \circ \exp \quad \text { for any } s \geq 0 \text { and any } t \geq s,
$$

where $\phi_{s, t}$ stands for $Q\left(\cdot, \omega_{t}\right) \circ \Phi_{s, t} \circ R\left(\cdot, \omega_{s}\right): \mathbb{S}_{r(s)} \rightarrow \mathbb{S}_{r(t)}$. Clearly it is sufficient to prove the following

Claim 1. For any closed interval $I:=[S, T] \subset[0,+\infty)$ and any compact set $K \subset$ $\mathbb{S}_{r(S)}$ there exists a non-negative function $k_{K, I} \in L^{d}(I, \mathbb{R})$ such that

$$
\left\|\phi_{s, t}-\phi_{s, u}\right\|_{K} \leq \int_{u}^{t} k_{K, I}(\xi) d \xi
$$

for any $s, u, t \in I$ such that $s \leq u \leq t$.

To prove the above claim we fix $I:=[S, T] \subset[0,+\infty)$ and a compact set $K \subset \mathbb{S}_{r(S)}$, and consider the set $K_{1}:=\bigcup_{s \in I} R\left(K, \omega_{s}\right)$. Since $R\left(\zeta, \omega_{s}\right)$ is jointly continuous in $\zeta$ and $s$ on $\mathbb{S}_{r(S)} \times I$, the set $K_{1} \subset \mathbb{H}$ is compact. Furthermore, it follows from Lemma 4.7 that $K_{2}:=\bigcup_{S \leq s \leq t \leq T} \Phi_{s, t}\left(K_{1}\right)$ is a compact set in $\mathbb{H}$. Finally, the function $Q(w, \omega)$ is holomorphic in a neighborhood of $K_{2} \times[0,+\infty)$ and the function $\tau \mapsto \omega_{\tau}$ is continuous on $I$. Hence there exists a constant $C_{1}=C_{1}\left(K_{2}, I\right)>0$ such that $\left\|Q\left(\cdot, \omega_{t}\right)-Q\left(\cdot, \omega_{u}\right)\right\|_{K_{2}} \leq C_{1}\left|\omega_{t}-\omega_{u}\right|$ for any $t, u \in I$. By the same reason there exists a constant $C_{2}=C_{2}\left(K_{2}, I\right)>0$ such that $\left\|Q\left(\cdot, \omega_{u}\right) \circ \Phi_{s, t}-Q\left(\cdot, \omega_{u}\right) \circ \Phi_{s, u}\right\|_{K_{1}} \leq C_{2}\left\|\Phi_{s, t}-\Phi_{s, u}\right\|_{K_{1}}$ for all $u \in I$.

Now we can estimate the left-hand side in (4.9) for any $s, u, t \in I, s \leq u \leq t$, as follows:

$$
\begin{gathered}
\left\|\phi_{s, t}-\phi_{s, u}\right\|_{K}=\left\|Q\left(\cdot, \omega_{t}\right) \circ \Phi_{s, t} \circ R\left(\cdot, \omega_{s}\right)-Q\left(\cdot, \omega_{u}\right) \circ \Phi_{s, u} \circ R\left(\cdot, \omega_{s}\right)\right\|_{K} \\
\leq\left\|Q\left(\cdot, \omega_{t}\right) \circ \Phi_{s, t}-Q\left(\cdot, \omega_{u}\right) \circ \Phi_{s, u}\right\|_{K_{1}} \leq\left\|Q\left(\cdot, \omega_{t}\right)-Q\left(\cdot, \omega_{u}\right)\right\|_{K_{2}} \\
+\left\|Q\left(\cdot, \omega_{u}\right) \circ \Phi_{s, t}-Q\left(\cdot, \omega_{u}\right) \circ \Phi_{s, u}\right\|_{K_{1}} \leq C_{1}\left|\omega_{t}-\omega_{u}\right|+C_{2}\left\|\Phi_{s, t}-\Phi_{s, u}\right\|_{K_{1}} .
\end{gathered}
$$

Thus our claim follows from assertion (ii) of Theorem 3.6 and from the fact that by Definition 4.1, $\tau \mapsto \omega_{\tau}=\omega(r(t))$ is of class $A C^{d}$. The proof is finished.

Proof of Proposition 4.5. By hypothesis, $\left(\varphi_{s, t}\right)$ is an evolution family of order $d$ over a canonical $L^{d}$-system $\left(D_{t}\right)$. Hence, obviously, it satisfies the conditions of Theorem 4.4. Therefore the statement of Claim 1 in the proof of Theorem 4.4 is true. Now Proposition 4.5 follows easily from (4.8), because the map $z \mapsto \exp z$ contracts the Euclidean metric in $\mathbb{S}_{r}$ for any $r \in[0,1)$.

Proof of Theorem 4.6. Let us first construct an $L^{d}$-evolution family $\left(\Psi_{s, t}\right)$ satisfying (4.2). Obviously, according to Lemma 4.8 the family $\left(\varphi_{s, t}\right)$ fulfills the conditions of Theorem 4.4. Hence by Lemma 4.10, there exists an $L^{d}$-evolution family $\left(\Phi_{s, t}\right)$ in $\mathbb{H}$ such that (4.5) holds for all $s \geq 0$ and all $t \geq s$. Recall that for each $\tau \geq 0$, 
$B_{\tau}=\exp \circ Q\left(\cdot, \omega_{\tau}\right)$, where $Q\left(\cdot, \omega_{\tau}\right)$ maps $\mathbb{H}$ conformally onto $\mathbb{S}_{r(\tau)}$. Therefore, bearing in mind $r(\tau)>0$ for any $\tau \geq 0$, we have $B_{\tau}(w)=W_{\tau}\left(Q\left(w, \omega_{\tau}\right) / \log r(\tau)\right)$ for all $\tau \geq 0$ and all $w \in \mathbb{H}$. It follows that the family $\left(\Psi_{s, t}\right)$, defined by

$$
\begin{gathered}
\quad \Psi_{s, t}:=P_{t} \circ \Phi_{s, t} \circ P_{s}^{-1}, \quad s \geq 0, t \geq s, \\
\text { where } \quad P_{\tau}(w):=\frac{Q\left(w, \omega_{\tau}\right)}{\log r(\tau)} \text { for all } w \in \mathbb{H} \text { and } \tau \geq 0,
\end{gathered}
$$

satisfies equality (4.2). It is also easy to see that $\left(\Psi_{s, t}\right)$ fulfills conditions EF1 and EF2. Hence, according to Proposition 3.7, to prove the existence statement of Theorem 4.6 it remains to check that for any $\zeta_{0} \in \mathbb{S}$ the function $[0,+\infty) \ni t \mapsto$ $\Psi_{0, t}(\zeta)$ belongs to $A C^{d}([0,+\infty), \mathbb{C})$.

Fix any $T>0$ and any $\zeta \in \mathbb{S}$. Denote $w:=P_{0}^{-1}(\zeta)$. Since $t \mapsto \Phi_{0, t}(w)$ is continuous, the set $K:=\left\{\Phi_{0, t}(w): t \in[0, T]\right\}$ is compact. Hence there exists a positive constant $C=C(\zeta, T)>0$ such that

$$
\left|P_{t}\left(w_{2}\right)-P_{u}\left(w_{1}\right)\right| \leq C\left(\left|w_{2}-w_{1}\right|+|r(t)-r(u)|\right)
$$

for all $w_{1}, w_{2} \in K$ and all $u, t \in[0, T]$. Therefore,

$$
\left|\Psi_{0, t}(\zeta)-\Psi_{0, u}(\zeta)\right| \leq C\left(|r(t)-r(u)|+\left|\Phi_{0, t}(w)-\Phi_{0, u}(w)\right|\right),
$$

whenever $u, t \in[0, T]$. The function $[0, T] \ni t \mapsto \Phi_{0, t}(w)$ is of class $A C^{d}$ by EF3, while $[0, T] \ni t \mapsto r(t)$ is of class $A C^{d}$ by definition. Together with the above inequality this means that $[0, T] \ni t \mapsto \Psi_{0, t}(\zeta)$ is also of class $A C^{d}$, which completes the proof of the fact that $\left(\Psi_{s, t}\right)$ is an $L^{d}$-evolution family in $\mathbb{S}$.

It remains to show the uniqueness of $\left(\Psi_{s, t}\right)$. To this end we fix an arbitrary $\zeta_{0} \in \mathbb{S}$ and take $w_{0}:=W_{0}\left(\zeta_{0}\right) \in \mathbb{A}_{r(0)}$. Now denote $w(t):=\varphi_{0, t}\left(w_{0}\right)$ and $\zeta(t):=\Psi_{0, t}\left(\zeta_{0}\right)$ for all $t \geq 0$. Then $W_{t}(\zeta(t))=w(t)$ for any $t \geq 0$. The mapping $W_{t}$ is a covering map of $\mathbb{S}$ onto $\mathbb{A}_{r(t)}$. Hence for any $s \geq 0$ and any $t \geq s$, the function $\Psi_{s, t}$ is the lifting of the mapping $\varphi_{s, t} \circ W_{s}$ w.r.t. $W_{t}$ that takes $\zeta(s)$ to $\zeta(t)$. It follows that the uniqueness of the family $\left(\Psi_{s, t}\right)$ is implied by the uniqueness of the continuous function $[0,+\infty) \ni t \mapsto \zeta(t) \in \mathbb{S}$ such that $\zeta(0)=\zeta_{0}$ and $W_{t}(\zeta(t))=w(t)$ for all $t \geq 0$. Such a function $t \mapsto \zeta(t)$ is unique because, according to the definition of $W_{t}$, the function $t \mapsto \zeta(t) \log r(t)$ is a lifting of $t \mapsto w(t)$ w.r.t. exp : $\mathbb{S}_{0} \rightarrow \mathbb{D}^{*}$. With the value $\zeta(0) \log r(0)$ being fixed, this completes the proof.

\section{Evolution FAMiLies AND DifFerential EQUATIONS}

This section contains our main results. We will establish a one-to-one correspondence between evolution families over canonical domain systems and semicomplete weak holomorphic vector fields, analogous to the correspondence between evolution families and Herglotz vector fields in the unit disk 10 and in complex hyperbolic manifolds [11. Moreover, we will give a precise constructive description of semicomplete weak holomorphic vector fields which appear in our setting for the non-degenerate case. At the end of the section we consider the degenerate case, which turns out to be reducible to the case of evolution families in the unit disk.

Throughout this section we fix arbitrary $d \in[1,+\infty]$ and some canonical domain system $\left(D_{t}\right):=\left(\mathbb{A}_{r(t)}\right)$ of order $d$. The set $\mathcal{D}:=\left\{(z, t): t \geq 0, z \in D_{t}\right\}$ is a 
relatively open subset of $\mathbb{C} \times E$, where $E:=[0,+\infty)$. So we can apply results of Section 2 to deduce the following

Theorem 5.1. The following two assertions hold.

(A) For any $L^{d}$-evolution family $\left(\varphi_{s, t}\right)$ over the canonical domain system $\left(D_{t}\right)$ there exists an essentially unique semicomplete weak holomorphic vector field $G: \mathcal{D} \rightarrow \mathbb{C}$ of order $d$ and a null-set $N \subset[0,+\infty)$ such that for all $s \geq 0$ the following statements hold:

(i) the mapping $[s,+\infty) \ni t \mapsto \varphi_{s, t} \in \operatorname{Hol}\left(D_{s}, \mathbb{C}\right)$ is locally absolutely continuous;

(ii) the mapping $[s,+\infty) \ni t \mapsto \varphi_{s, t} \in \operatorname{Hol}\left(D_{s}, \mathbb{C}\right)$ is differentiable for all $t \in[s,+\infty) \backslash N$

(iii) $d \varphi_{s, t} / d t=G(\cdot, t) \circ \varphi_{s, t}$ for all $t \in[s,+\infty) \backslash N$.

(B) For any semicomplete weak holomorphic vector field $G: \mathcal{D} \rightarrow \mathbb{C}$ of order $d$ the formula $\varphi_{s, t}(z):=w_{s}^{*}(z, t), t \geq s \geq 0, z \in D_{s}$, where $w_{s}^{*}(z, \cdot)$ is the unique maximal solution to the initial value problem

$$
\dot{w}=G(w, t), \quad w(s)=z,
$$

defines an $L^{d}$-evolution family over the canonical domain system $\left(D_{t}\right)$.

In the situation of the above theorem we will say that $G$ is the vector field corresponding to the evolution family $\left(\varphi_{s, t}\right)$. The phrase essentially unique in this theorem means that for any two vector fields $G_{1}$ and $G_{2}$ corresponding to the same evolution family, $G_{1}(\cdot, t)=G_{2}(\cdot, t)$ for a.e. $t \geq 0$.

Proof. Assertion (B) of the theorem follows readily from Proposition 2.6. According to Theorem 2.10, in order to prove (A), we only have to check that assertions (i)(iv) of Proposition 2.6 hold for any $L^{d}$-evolution family $\left(\varphi_{s, t}\right)$ over $\left(D_{t}\right)$. The first three of them hold by the very definition of an evolution family over a canonical domain system. To prove assertion (iv) of Proposition 2.6 we fix any compact set $K \subset \mathcal{D}$ and any $T>\operatorname{maxpr}_{\mathbb{R}}(K)$. Since $K$ is compact, there exist finite sequences $\left(S_{j}\right)_{j=1}^{n} \subset[0, T]$ and $\left(K_{j}\right)_{j=1}^{n}$ such that $K_{j} \subset D_{S_{j}}$ is a compact set for each $j=1, \ldots, n$ and $K \subset \bigcup_{j=1}^{n} K_{j} \times\left[S_{j}, T\right]$. Now apply Proposition 4.5 with $K_{j}$ and $I_{j}:=\left[S_{j}, T\right]$ substituted for $K$ and $I$, respectively. Further, extend the function $k_{K_{j}, I_{j}}$ by zero to $\left[0, S_{j}\right)$ and define $k_{K}^{T}:=\sum_{j=1}^{n} k_{K_{j}, I_{j}} \in L^{d}([0, T], \mathbb{R})$. Then, for any $(z, s) \in K$ and any $u, t \in[s, T]$ with $u \leq t$,

$$
\left|\varphi_{s, t}(z)-\varphi_{s, u}(z)\right| \leq \int_{u}^{t} k_{K}^{T}(\xi) d \xi .
$$

Take any increasing sequence $\left(T_{n}\right)$ such that $T_{n}>\max \operatorname{pr}_{\mathbb{R}}(K)$ for all $n \in \mathbb{N}$ and $T_{n} \rightarrow+\infty$ as $n \rightarrow+\infty$. Now we finish the proof of assertion (iv) of Proposition 2.6 by setting $\tilde{k}_{K}:=k_{K}^{T_{1}} \chi_{\left[0, T_{1}\right)}+\sum_{n=2}^{+\infty} k_{K}^{T_{n}} \chi_{\left[T_{n-1}, T_{n}\right)}$.

Finally, the fact that $G$ is essentially unique follows from statement (iii) of the theorem with $s:=t$. The proof is finished.

5.1. Semicomplete weak holomorphic vector fields in non-degenerate case. In this subsection we are going to give a precise constructive description of semicomplete weak holomorphic vector fields in $\mathcal{D}:=\left\{(z, t): t \geq 0, z \in D_{t}\right\}$ for the 
case when the canonical domain system $\left(D_{t}\right)$ is non-degenerate, i.e., when $r(t)>0$ for all $t \geq 0$.

In order to state the main result of the subsection we need some notation. First of all, for $r>0$ we denote by $\mathcal{K}_{r}: \mathbb{A}_{r} \rightarrow \mathbb{C}$ the so-called Villat kernel, which can be defined by the following formula (see, e.g., [21] or [4, $\S \mathrm{V} .1]$ ):

$$
\mathcal{K}_{r}(z):=\lim _{n \rightarrow+\infty} \sum_{\nu=-n}^{n} \frac{1+r^{2 \nu} z}{1-r^{2 \nu} z}=\frac{1+z}{1-z}+\sum_{\nu=1}^{+\infty}\left(\frac{1+r^{2 \nu} z}{1-r^{2 \nu} z}+\frac{1+z / r^{2 \nu}}{1-z / r^{2 \nu}}\right) .
$$

The Villat kernel plays the same role for the function theory in the annulus as the Schwartz kernel $\mathcal{K}_{0}(z):=(1+z) /(1-z)$ in the unit disk. Namely, for any function $f \in \operatorname{Hol}\left(\mathbb{A}_{r}, \mathbb{C}\right)$ which is continuous in $\overline{\mathbb{A}_{r}}$ (see, e.g., [43, Theorem 2.2.10]),

$$
\begin{aligned}
f(z)=\int_{\mathbb{T}} \mathcal{K}_{r}\left(z \xi^{-1}\right) \operatorname{Re} f(\xi) & \frac{|d \xi|}{2 \pi}+\int_{\mathbb{T}}\left[\mathcal{K}_{r}(r \xi / z)-1\right] \operatorname{Re} f(r \xi) \frac{|d \xi|}{2 \pi} \\
& +i \int_{\mathbb{T}} \operatorname{Im} f(\rho \xi) \frac{|d \xi|}{2 \pi} \quad \text { for all } z \in \mathbb{A}_{r}, \rho \in[r, 1] .
\end{aligned}
$$

Remark 5.2. Fix any $r \in(0,1)$ and $\rho \in(r, 1)$. It is known (see, e.g., [21] or [4, $\S \mathrm{V} .1])$ that

$$
\max _{\theta \in \mathbb{R}} \operatorname{Re} \mathcal{K}_{r}\left(e^{i \theta} \rho\right)=\mathcal{K}_{r}(\rho)>1, \quad \min _{\theta \in \mathbb{R}} \operatorname{Re} \mathcal{K}_{r}\left(e^{i \theta} \rho\right)=\mathcal{K}_{r}(-\rho) \in(0,1) .
$$

Moreover, $\mathcal{K}_{r}(x)$ is increasing on $[-1,-r]$ and on $[r, 1)$ with $\mathcal{K}_{r}( \pm r)=1$ and $\mathcal{K}_{r}(-1)=0$.

Definition 5.3. Let $r \in(0,1)$. By the class $\mathcal{V}_{r}$ we will mean the collection of all functions $p \in \operatorname{Hol}\left(\mathbb{A}_{r}, \mathbb{C}\right)$ having the integral representation

$$
p(z)=\int_{\mathbb{T}} \mathcal{K}_{r}(z / \xi) d \mu_{1}(\xi)+\int_{\mathbb{T}}\left[1-\mathcal{K}_{r}(r \xi / z)\right] d \mu_{2}(\xi), \quad z \in \mathbb{A}_{r},
$$

where $\mu_{1}$ and $\mu_{2}$ are positive Borel measures on the unit circle $\mathbb{T}$ subject to the condition $\mu_{1}(\mathbb{T})+\mu_{2}(\mathbb{T})=1$.

Remark 5.4. Given $p \in \mathcal{V}_{r}$, the measures $\mu_{1}$ and $\mu_{2}$ in the representation formula (5.4) are unique. This statement follows easily from the proof of [46, Theorem 1], which we will briefly discuss later in the paper. Another way to show the uniqueness of $\mu_{1}$ and $\mu_{2}$ in (5.4) is based on the fact that there do not exist signed Borel measures $m_{1}$ and $m_{2}$ on $\mathbb{T}$ such that $\left|m_{1}\right|(\mathbb{T}),\left|m_{2}\right|(\mathbb{T})<+\infty,\left|m_{1}\right|(\mathbb{T})+\left|m_{2}\right|(\mathbb{T}) \neq 0$ (where $|\cdot|$ stands for the variation of a measure), $m_{1}(\mathbb{T})+m_{2}(\mathbb{T})=0$, and for all $z \in \mathbb{A}_{r}$

$$
\mathcal{J}_{m_{1}}(z)+m_{2}(\mathbb{T})-\mathcal{J}_{m_{2}}(r / z)=0, \quad \mathcal{J}_{m_{j}}(z):=\int_{\mathbb{T}} \mathcal{K}_{r}(z / \xi) d m_{j}(\xi), j=1,2 .
$$

Indeed, passing to the real part in (5.5) and taking into consideration that $\mathcal{K}_{r}\left(\rho e^{i \theta}\right)-$ $\mathcal{K}_{0}\left(\rho e^{i \theta}\right) \rightarrow 0$ uniformly w.r.t. $\theta \in \mathbb{R}$ as $\rho \rightarrow 1-0$ and $\operatorname{Re} \mathcal{K}_{r}\left(\rho e^{i \theta}\right) \rightarrow 1$ uniformly w.r.t. $\theta \in \mathbb{R}$ as $\rho \rightarrow r+0$, one may conclude that the Fourier series $\sum_{k \in \mathbb{Z}} c_{1, k} e^{i \theta k}$ of the measure $m_{1}$ is Abel summable to zero and moreover, $\sum_{k \in \mathbb{Z}} \rho^{k} c_{1, k} e^{i \theta k} \rightarrow 0$ uniformly w.r.t. $\theta$ as $\rho \rightarrow 1-0$. With the Fourier coefficients of $m_{1}$ being bounded, it follows that $c_{1, k}=0$ for all $k \in \mathbb{Z}$ and thus $m_{1}=0$. As a consequence, $m_{2}(\mathbb{T})=0$ and we can finally deduce from (5.5) that $m_{2}=0$ in an analogous way. 
Let $F \in \operatorname{Hol}\left(\mathbb{A}_{r}, \mathbb{C}\right)$ for some $r \in(0,1)$. Denote by $\mathcal{N}(F)$ the free term in the Laurent expansion of $F$,

$$
\mathcal{N}(F):=\int_{\mathbb{T}} F(\rho \xi) \frac{|d \xi|}{2 \pi}, \quad \rho \in(r, 1) .
$$

Remark 5.5. Since $\mathcal{N}\left(\mathcal{K}_{r}\right)=1$, we have $\mathcal{N}(p)=\mu_{1}(\mathbb{T}) \geq 0$ for any $p \in \mathcal{V}_{r}$.

Now we can formulate the main result of this subsection.

Theorem 5.6. Let $d \in[1,+\infty]$, and let $\left(D_{t}\right)=\left(\mathbb{A}_{r(t)}\right)$ be a non-degenerate canonical domain system of order $d$. Then a function $G: \mathcal{D} \rightarrow \mathbb{C}$, where $\mathcal{D}:=\{(z, t)$ : $\left.t \geq 0, z \in D_{t}\right\}$, is a semicomplete weak holomorphic vector field of order $d$ if and only if there exist functions $p: \mathcal{D} \rightarrow \mathbb{C}$ and $C:[0,+\infty) \rightarrow \mathbb{R}$ such that:

(i) $G(w, t)=w\left[i C(t)+r^{\prime}(t) p(w, t) / r(t)\right]$ for a.e. $t \geq 0$ and all $w \in D_{t}$;

(ii) for each $w \in D:=\bigcup_{t \geq 0} D_{t}$ the function $p(w, \cdot)$ is measurable in $E_{w}:=$ $\{t \geq 0:(w, t) \in \mathcal{D}\}$

(iii) for each $t \geq 0$ the function $p(\cdot, t)$ belongs to the class $\mathcal{V}_{r(t)}$;

(iv) $C \in L_{\text {loc }}^{d}([0,+\infty), \mathbb{R})$.

The following proposition forms the main block for the proof of sufficiency in Theorem 5.6.

Proposition 5.7. Let $\left(D_{t}\right)=\left(\mathbb{A}_{r(t)}\right)$ be a canonical domain system of order $d \in$ $[1,+\infty]$, and let $G$ be a semicomplete weak holomorphic vector field in $\mathcal{D}:=\{(z, t)$ : $\left.t \geq 0, z \in D_{t}\right\}$ of the same order. Then, for a.e. $t \in E:=\{t \geq 0: r(t)>0\}$, the function $G_{t}:=G(\cdot, t): D_{t} \rightarrow \mathbb{C}$ admits the representation

$$
G_{t}(z)=z\left(\frac{r^{\prime}(t)}{r(t)} p_{t}(z)+i C_{t}\right), \quad C_{t} \in \mathbb{R}, p_{t} \in \mathcal{V}_{r(t)}
$$

Remark 5.8. Denote by $p\left[r, \mu_{1}, \mu_{2}\right]$ the function defined by representation (5.4) and let $\widehat{\mu}$ stand for the push-forward of a Borel measure $\mu$ on $\mathbb{T}$ w.r.t. the map $z \mapsto \bar{z}$. Let $r \in(0,1)$ and $p \in \mathcal{V}_{r}$. By definition, $p=p\left[r, \mu_{1}, \mu_{2}\right]$ for some positive Borel measures $\mu_{1}$ and $\mu_{2}$ on $\mathbb{T}$ subject to the condition $\mu_{1}(\mathbb{T})+\mu_{2}(\mathbb{T})=1$. It is easy to see that the function $\tilde{p}(z):=1-p(r / z), z \in \mathbb{A}_{r}$, also belongs to $\mathcal{V}_{r}$ and $\tilde{p}=p\left[r, \widehat{\mu}_{2}, \widehat{\mu}_{1}\right]$.

Denote by $\mathcal{C}$ the Carathéodory class of all functions $q \in \operatorname{Hol}(\mathbb{D}, \mathbb{C})$ normalized by the condition $q(0)=1$ and satisfying for all $z \in \mathbb{D}$ the inequality $\operatorname{Re} q(z) \geq 0$. This class has many nice properties, one of which can be formulated as follows.

Remark 5.9. The class $\mathcal{C}$ is a compact convex subset of $\mathrm{Hol}(\mathbb{D}, \mathbb{C})$. Moreover, for any continuous convex functional $L: \mathcal{C} \rightarrow \mathbb{R}$,

$$
\max _{q \in \mathcal{C}} L(q)=\max _{\theta \in \mathbb{R}} L\left(\mathcal{K}_{0}^{\theta}\right), \quad \text { where } \mathcal{K}_{0}^{\theta}(z):=\mathcal{K}_{0}\left(z e^{-i \theta}\right) .
$$

Indeed, to prove the above statement one only has to apply the Krein-Milman theorem (see, e.g., [38, p. 181]) and take into account that the set of all extremal points of $\mathcal{C}$ coincides with $\left\{\mathcal{K}_{0}^{\theta}: \theta \in \mathbb{R}\right\}$ (see, e.g., [24]).

Our proof of Proposition 5.7 takes advantage of the following lemmas. Recall the notation $\mathbb{S}:=\{w: 0<\operatorname{Re} w<1\}$. 
Lemma 5.10. Let $p \in \operatorname{Hol}(\mathbb{S}, \mathbb{C})$. Suppose that $\operatorname{Re} p(w) \geq 0$ for all $w \in \mathbb{S}$. Then for any $a, b \in \mathbb{R}, a<b$,

(i) there exists $C_{1}(a, b, p)>0$ such that

$$
L_{2}(p, u):=\int_{a}^{b}|\operatorname{Im} p(u+i v)| d v \leq-C_{2}(a, b, p) \log [u(1-u)], \quad \text { for all } u \in(0,1) .
$$

Proof. Fix any $a, b \in \mathbb{R}, a>b$. Note that

$L_{1}(p, u)=\operatorname{Re} p(1 / 2) L_{1}\left(p_{0}, u\right)$ and $L_{2}(p, u) \leq(b-a)|\operatorname{Im} p(1 / 2)|+\operatorname{Re} p(1 / 2) L_{2}\left(p_{0}, u\right)$ for any $u \in(0,1)$, where $p_{0}(w):=(p(w)-i \operatorname{Im} p(1 / 2)) / \operatorname{Re} p(1 / 2)$ if $\operatorname{Re} p(1 / 2)>0$ and $p_{0} \equiv 1$ if $\operatorname{Re} p(1 / 2)=0$.

Therefore, it is sufficient to prove the lemma only for functions $p \in \operatorname{Hol}(\mathbb{S}, \mathbb{C})$ satisfying $\operatorname{Re} p(w) \geq 0$ for all $w \in \mathbb{S}$ and normalized by $p(1 / 2)=1$. So, we further suppose that $p$ fulfills these conditions.

Let $F$ stand for the conformal mapping of the unit disk $\mathbb{D}$ onto the strip $\mathbb{S}$ normalized by $F(0)=1 / 2, i F^{\prime}(0)>0$, namely

$$
F(z)=\frac{1}{\pi i} \log \left(i \frac{1+z}{1-z}\right) .
$$

Consider the function $q:=p \circ F$. It belongs to the Carathéodory class $\mathcal{C}$. For each fixed $u \in(0,1)$ the functionals $q \mapsto L_{1}^{\mathbb{D}}(q):=L_{1}\left(q \circ F^{-1}, u\right)$ and $q \mapsto L_{2}^{\mathbb{D}}(q):=$ $L_{2}\left(q \circ F^{-1}, u\right)$ are convex on the class $\mathcal{C}$. Therefore, according to Remark 5.9 we can restrict ourselves to the case $q=\mathcal{K}_{0}^{\theta}, \theta \in \mathbb{R}$. In this case $p=p_{\theta}:=\mathcal{K}_{0}^{\theta} \circ F^{-1}$.

Let us fix $\varepsilon \in(0,1 / 2)$. Since the functions $u \mapsto L_{j}(p, u), j=1,2$, are continuous for $u \in(0,1)$, it is sufficient to prove (i) and (ii) for all $u \in U_{\varepsilon}:=(0,1 / 2-\varepsilon) \cup$ $(1 / 2+\varepsilon, 1)$. Denote $I(u):=F^{-1}(\{w=u+i v: v \in[a, b]\})$. The function $F$ extends holomorphically to the closed unit disk minus $\{ \pm 1\}$. For any $u \in U_{\varepsilon}$ the preimage of the straight line $\{u+i v: v \in \mathbb{R}\}$ under $F$ is a circular arc joining points \pm 1 , while the preimages of the segments $[i a, 1+i a]$ and $[i b, 1+i b]$ are the hyperbolic geodesics symmetric w.r.t. the real line. In particular, it follows that there exists $A(a, b, \varepsilon)>0$ such that for any $u \in U_{\varepsilon},|d F(z)| /|d \arg z|<A(a, b, \varepsilon)$ when $z$ moves along $I(u)$. Hence,

$$
\begin{aligned}
& L_{1}\left(p_{\theta}, u\right) \leq A(a, b, \varepsilon) \int_{I(u)} \operatorname{Re} \mathcal{K}_{0}\left(z e^{-i \theta}\right)|d \arg z|, \\
& L_{2}\left(p_{\theta}, u\right) \leq A(a, b, \varepsilon) \int_{I(u)}\left|\operatorname{Im} \mathcal{K}_{0}\left(z e^{-i \theta}\right)\right||d \arg z|
\end{aligned}
$$

for all $u \in U_{\varepsilon}$ and all $\theta \in \mathbb{R}$.

Again using properties of the function $F$, we conclude that there are positive constants $B_{1}(a, b)$ and $B_{2}(a, b)$ not depending on $u$ such that

$$
\rho_{1}(u):=1-B_{1}(a, b) u(1-u) \leq|z| \leq 1-B_{2}(a, b) u(1-u)=: \rho_{2}(u)
$$

for all $u \in U_{\varepsilon}$ and all $z \in I(u)$. 
Recall that

$$
\mathcal{P}_{0}(\rho, \alpha):=\operatorname{Re} \mathcal{K}_{0}\left(\rho e^{i \alpha}\right)=\frac{1-\rho^{2}}{1+\rho^{2}-2 \rho \cos \alpha} .
$$

Hence, according to (5.12) the integrand on the right-hand side of (5.10) can be estimated as

$$
\operatorname{Re} \mathcal{K}_{0}\left(z e^{-i \theta}\right) \leq \mathcal{P}_{0}\left(\rho_{2}(u),-\theta+\arg z\right) \frac{1-\rho_{1}(u)}{1-\rho_{2}(u)}=\mathcal{P}_{0}\left(\rho_{2}(u),-\theta+\arg z\right) \frac{B_{1}(a, b)}{B_{2}(a, b)}
$$

for all $z \in I(u)$. It follows that the integral itself is not greater than

$$
\int_{0}^{2 \pi} \mathcal{P}_{0}\left(\rho_{2}(u),-\theta+\alpha\right) \frac{B_{1}(a, b)}{B_{2}(a, b)} d \alpha=2 \pi \frac{B_{1}(a, b)}{B_{2}(a, b)} .
$$

This proves statement (i).

Statement (ii) can be proved in a similar way if one notices that for each fixed $\alpha \in \mathbb{R}$ the function $\rho \mapsto\left|\operatorname{Im} \mathcal{K}_{0}\left(\rho e^{i \alpha}\right)\right|$ is non-decreasing on $(0,1)$ and that

$$
\int_{0}^{2 \pi}\left|\operatorname{Im} \mathcal{K}_{0}\left(\rho e^{i \alpha}\right)\right| d \alpha=4 \log \frac{1+\rho}{1-\rho} .
$$

Lemma 5.11. Let $r \in(0,1)$ and $F \in \operatorname{Hol}\left(\mathbb{A}_{r}, \mathbb{C}\right)$. Suppose that there exist constants $\alpha \geq 0, w_{0} \in \overline{\mathbb{S}} \backslash\{\infty\}$ and a function $p \in \operatorname{Hol}(\mathbb{S}, \mathbb{C})$ such that for all $w \in \mathbb{S}$,

$$
\operatorname{Re} p(w) \geq 0 \text { and } F(W(w))=\alpha w+p(w) \sin \frac{\pi}{2}\left(w_{0}-w\right) \sin \frac{\pi}{2}\left(\bar{w}_{0}+w\right),
$$

where $W(w):=\exp (w \log r)$. Then

$$
F(z)=i C+\alpha\left[\int_{\mathbb{T}} \mathcal{K}_{r}\left(z \xi^{-1}\right) d \mu_{1}(\xi)+\int_{\mathbb{T}}\left[1-\mathcal{K}_{r}(r(t) \xi / z)\right] d \mu_{2}(\xi)\right], \quad z \in \mathbb{A}_{r},
$$

for some constant $C \in \mathbb{R}$ and positive Borel measures $\mu_{1}$ and $\mu_{2}$ on the unit circle $\mathbb{T}$ subject to the condition $\mu_{1}(\mathbb{T})+\mu_{2}(\mathbb{T})=1$.

Proof. Let us prove first that

$$
\int_{\mathbb{T}}|\operatorname{Re} F(\rho \xi)||d \xi|<M<+\infty
$$

for all $\rho \in(r, 1)$ and some constant $M>0$ not depending on $\rho$.

Write $\kappa(w):=\sin \frac{\pi}{2}\left(w_{0}-w\right) \sin \frac{\pi}{2}\left(\bar{w}_{0}+w\right)$. Since

$$
\operatorname{Re} F(W(w))=\alpha \operatorname{Re} w+\operatorname{Re} \kappa(w) \operatorname{Re} p(w)-\operatorname{Im} \kappa(w) \operatorname{Im} p(w),
$$

we can estimate the integral in (5.14) from above by the sum $I_{1}(u)+I_{2}(u)+I_{3}(u)$, where

$$
\begin{gathered}
I_{1}(u):=\int_{0}^{T} \alpha \operatorname{Re}(u+i v) d v=\alpha u T, I_{2}(u):=\int_{0}^{T}|\operatorname{Re} \kappa(u+i v) \operatorname{Re} p(u+i v)| d v, \\
I_{3}(u):=\int_{0}^{T}|\operatorname{Im} \kappa(u+i v) \operatorname{Im} p(u+i v)| d v
\end{gathered}
$$

where $u:=\log \rho / \log r \in(0,1), T:=2 \pi /|\log r|$. 
Now we note that if $w_{0}=u_{0}+i v_{0}$, then

$$
\begin{gathered}
\operatorname{Re} \kappa(u+i v)=\frac{1}{2}\left[-\cos \pi u_{0}+\cos \pi u \operatorname{ch} \pi\left(v_{0}-v\right)\right], \\
\operatorname{Im} \kappa(u+i v)=\frac{1}{2} \sin \pi u \operatorname{sh} \pi\left(v_{0}-v\right) .
\end{gathered}
$$

In particular, $\operatorname{Re} \kappa(u+i v)$ is bounded for $u \in(0,1)$ and $v \in[0, T]$, while for $\operatorname{Im} \kappa$ we have

$$
|\operatorname{Im} \kappa(u+i v)| \leq 2 u(1-u)\left|\operatorname{sh} \pi\left(v_{0}-v\right)\right| \leq e^{\pi\left|v-v_{0}\right|} u(1-u) .
$$

Therefore, using Lemma 5.10 we conclude that $I_{2}(u)$ is a bounded function of $u \in(0,1)$ and that $I_{3}(u) \rightarrow 0$ as $u \rightarrow 1-0$ and as $u \rightarrow+0$. This proves (5.14). Then the following Villat-Stieltjes representation [46, Theorem 1] takes place:2]

$$
F(z)=i C+\int_{\mathbb{T}} \mathcal{K}_{r}\left(z \xi^{-1}\right) d \nu_{1}(\xi)+\int_{\mathbb{T}}\left[\mathcal{K}_{r}(r \xi / z)-1\right] d \nu_{2}(\xi), \quad z \in \mathbb{A}_{r},
$$

where $\nu_{k}, k=1,2$, are finite signed Borel measures on $\mathbb{T}$ with $\nu_{1}(\mathbb{T})=\nu_{2}(\mathbb{T})$, and $C \in \mathbb{R}$.

The proof of (5.18), given by Zmorovič in 46, is similar to the proof of the Herglotz representation theorem, which can be found in [18, §1.9]. Namely, for $\rho \in(r, 1)$ and $t \in[0,2 \pi]$ we write

$$
\nu_{\rho}^{ \pm}(t):=\frac{1}{2 \pi} \int_{0}^{t} F^{ \pm}(\rho, \theta) d \theta, \quad \text { where } F^{ \pm}(\rho, \theta):=\frac{\left|\operatorname{Re} F\left(\rho e^{i \theta}\right)\right| \pm \operatorname{Re} F\left(\rho e^{i \theta}\right)}{2} .
$$

The functions $\nu_{\rho}^{+}$and $\nu_{\rho}^{-}, \rho \in(0,1)$, are non-decreasing and, by (5.14), are uniformly bounded. Hence applying the Helly selection theorem (see, e.g., [18, p. 22]), we conclude that there is a sequence $\left(\rho_{n}\right) \subset(\sqrt{r}, 1)$ converging to 1 and two signed measures $\nu_{j}, j=1,2$, on $\mathbb{T}$ such that $d \nu_{\rho_{j, n}}^{+}(\arg \xi)-d \nu_{\rho_{j, n}}^{-}(\arg \xi) \rightarrow d \nu_{j}(\xi)$ in the sense of weak-* convergence as $n \rightarrow+\infty$, where $\rho_{1, n}:=\rho_{n}, \rho_{2, n}:=r / \rho_{n}$. It follows, in particular, that $\nu_{1}(\mathbb{T})=\nu_{2}(\mathbb{T})$, because $\nu_{\rho}^{+}(2 \pi)-\nu_{\rho}^{-}(2 \pi)$ does not depend on $\rho$ according to the Cauchy integral theorem. To see that $\nu_{j}$ satisfies (5.18), Zmorovič used the Villat formula (5.3) to represent the function $F$ in the annulus $A_{n}:=\left\{z: \rho_{2, n}<|z|<\rho_{1, n}\right\}$ via the values of its real part on $\partial A_{n}$. The weak-* convergence mentioned above means that for any continuous function $\varphi$ on $\mathbb{T}$,

$$
\int_{\mathbb{T}} \varphi d \nu_{j}=\lim _{n \rightarrow+\infty} \int_{\mathbb{T}} \varphi(\xi) \operatorname{Re} F\left(\rho_{j, n} \xi\right) d m(\xi), \quad j=1,2,
$$

where $m$ denotes the normalized standard Lebesgue measure on $\mathbb{T}, d m(\xi)$ $=|d \xi| /(2 \pi)$.

Now consider the integral $J(u):=\int_{0}^{T} \varphi\left(e^{i v \log r}\right) \operatorname{Re} p(u+i v) \operatorname{Re} \kappa(u+i v) d v$, where $\varphi: \mathbb{T} \rightarrow[0,1]$ is a continuous function. Taking into account (5.7), for each

\footnotetext{
${ }^{2}$ The proof of the result we refer to seems to be published only in Russian; for the formulation of this result in English, see the Zentralblatt review of [46], Zbl 0074.05701. Earlier Komatu [26] obtained the Villat-Stieltjes representation in a different form, involving the Weierstraß zetafunction. Connection between these two forms can be seen using an expansion of the Weierstraß zeta-function given, e.g., in [3] Ch.IV $\S 20]$.
} 
$u \in(0,1)$ we have

$$
\begin{aligned}
C_{1}(0, T) M_{1}(u) \leq & M_{1}(u) \int_{0}^{T} \operatorname{Re} p(u+i v) d v \\
& \leq J(u) \leq M_{2}(u) \int_{0}^{T} \operatorname{Re} p(u+i v) d v \leq C_{1}(0, T) M_{2}(u),
\end{aligned}
$$

where $M_{1}(u):=\min \{0, \operatorname{Re} \kappa(u+i v): v \in[0, T]\}$ and $M_{2}(u):=\max \{0, \operatorname{Re} \kappa(u+i v):$ $v \in[0, T]\}$.

Since $I_{1}(u), I_{3}(u)$ and $M_{1}(u)$ tend to 0 as $u \rightarrow+0$, we deduce from (5.19) with $j:=1$ and (5.20) that $\int_{\mathbb{T}} \varphi d \nu_{1} \geq 0$ for any continuous $\varphi: \mathbb{T} \rightarrow[0,1]$. It follows that $\nu_{1} \geq 0$.

Analogously, using (5.19) with $j:=2$, we prove that $\nu_{2} \leq \alpha m$. Since $\nu_{1}(\mathbb{T})=$ $\nu_{2}(\mathbb{T})$, we can conclude that $\nu_{1}(\mathbb{T}) \in[0, \alpha]$. Set $\mu_{1}:=\nu_{1} / \alpha$ and $\mu_{2}:=m-\nu_{2} / \alpha$. Clearly, $\mu_{1}$ and $\mu_{2}$ are positive Borel measures on $\mathbb{T}$ and $\mu_{1}(\mathbb{T})+\mu_{2}(\mathbb{T})=1$. Now let us notice that the second integral in (5.18) does not change in value if one adds to the measure $\nu_{2}$ any constant multiple of $m$, because $\int_{\mathbb{T}} \mathcal{K}_{r}(\rho \xi) d m(\xi)=1$ for any $\rho \in(r, 1)$. With this remark one immediately obtains representation (5.13) from (5.18). The proof is finished.

Lemma 5.12. Let $p \in \operatorname{Hol}(\mathbb{S}, \mathbb{C})$ and $\operatorname{Re} p(w) \geq 0$ for all $w \in \mathbb{S}$. Then there exist finite non-negative limits

$$
A:=\lim _{v \rightarrow+\infty} e^{-\pi v} p(1 / 2+i v), \quad B:=\lim _{v \rightarrow-\infty} e^{\pi v} p(1 / 2+i v) .
$$

Proof. For $z$ in the upper half-plane $\mathbb{H}:=\{z: \operatorname{Im} z>0\}$ write

$$
p_{1}(z):=i p\left(\frac{\log z}{i \pi}\right), \quad p_{2}(z):=p_{1}(-1 / z),
$$

where the branch of $\log$ is chosen by setting $\log i=i \pi / 2$. Then the limits in the statement of the lemma will take the form

$$
A=\lim _{y \rightarrow+\infty} \frac{p_{2}(i y)}{i y}, \quad B=\lim _{y \rightarrow+\infty} \frac{p_{1}(i y)}{i y} .
$$

Notice that $p_{1}, p_{2} \in \mathrm{Hol}(\mathbb{H}, \overline{\mathbb{H}})$ and apply the classical result about existence of the angular derivative at $\infty$; see, e.g., [44, Ch. IV Sect.26].

Proof of Proposition 5.7. For simplicity we assume that $r(t)>0$ for all $t \geq 0$. However, the argument below can be easily adapted for the general case of a canonical domain system $\left(D_{t}\right)$ of mixed type.

By Theorem 5.1 (iv) there exists an $L^{d}$-evolution family over $\left(D_{t}\right)$ and a null-set $N_{1} \subset[0,+\infty)$ such that for every $s \geq 0$,

$$
\frac{d \varphi_{s, t}(z)}{d t}=G_{t}\left(\varphi_{s, t}(z)\right) \quad \text { for all } z \in D_{s} \text { and all } t \in[s,+\infty) \backslash N_{1} .
$$

By Theorem 4.6 there exists an $L^{d}$-evolution family $\left(\Psi_{s, t}\right)$ in $\mathbb{S}$ such that $\varphi_{s, t} \circ W_{s}=$ $W_{t} \circ \Psi_{s, t}$ for all $s \geq 0$ and all $t \geq s$, where $W_{\tau}(w):=\exp (w \log r(\tau))$ for all $\tau \geq 0$ and all $w \in \mathbb{S}$. Furthermore by Theorem [3.6. there exists a null-set $N_{2} \subset[0,+\infty)$ such that for every $s \geq 0$,

$$
\frac{d \Psi_{s, t}(w)}{d t}=\tilde{G}_{t}\left(\Psi_{s, t}(w)\right) \quad \text { for all } w \in \mathbb{S} \text { and all } t \in[s,+\infty) \backslash N_{2},
$$


where $\tilde{G}_{t}: \mathbb{S} \rightarrow \mathbb{C}$ is an infinitesimal generator for each $t \in[0,+\infty) \backslash N_{2}$. Finally, the mapping $t \mapsto r(t)$ is of class $A C^{d}$. Hence $t \mapsto \log r(t)$ is differentiable for all $t \geq 0$ aside some null-set $N_{3}$. Thus setting in the above equalities $z:=W_{s}(w)$ and then letting $s:=t$, we conclude that for all $t \in[0,+\infty) \backslash N$, where $N:=N_{1} \cup N_{2} \cup N_{3}$, and all $w \in \mathbb{S}$,

$$
G_{t}\left(W_{t}(w)\right)=W_{t}(w)\left[w \frac{r^{\prime}(t)}{r(t)}+\tilde{G}_{t}(w) \log r(t)\right] .
$$

Now to show that $G_{t}$ is of form (5.6), we fix any $t \in[0,+\infty) \backslash N$ and take advantage of the Berkson-Porta representation for infinitesimal generators in the unit disk $\mathbb{D}$. Namely, according to Remark 3.4 with $F$ given by (5.9), $\tilde{G}_{t}(w)=$ $F^{\prime}\left(F^{-1}(w)\right) H\left(F^{-1}(w)\right)$ for all $w \in \mathbb{S}$, where $F^{-1}(w)=i \operatorname{tg}(\pi(w-1 / 2) / 2)$ and $H(z):=(\tau-z)(1-\bar{\tau} z) p(z)$ for some point $\tau \in \overline{\mathbb{D}}$ and some function $p \in \operatorname{Hol}(\mathbb{D}, \mathbb{C})$ satisfying $\operatorname{Re} p(z) \geq 0$ for all $z \in \mathbb{D}$. Writing $w_{0}:=F^{-1}(\tau)$, we finally get that either

$$
\tilde{G}_{t}(w)=\sin \left[\pi\left(\bar{w}_{0}+w\right) / 2\right] \sin \left[\pi\left(w_{0}-w\right) / 2\right] \tilde{p}_{t}(w), \quad w_{0} \in \overline{\mathbb{S}} \backslash\{\infty\},
$$

if $\tau \in \overline{\mathbb{D}} \backslash\{ \pm 1\}$, or

$$
\tilde{G}_{t}(w)=e^{ \pm i \pi w} \tilde{p}_{t}(w) \text { if } \tau= \pm 1,
$$

where in both cases $\tilde{p}_{t} \in \operatorname{Hol}(\mathbb{S}, \mathbb{C})$ and $\operatorname{Re} \tilde{p}_{t}(w) \geq 0$ for all $w \in \mathbb{S}$.

Assume first that equality (5.22) takes place. Applying Lemma 5.11 for $r:=$ $r(t), F(z):=-G_{t}(z) / z, \alpha:=-r^{\prime}(t) / r(t)$ and $p(w):=-\tilde{p}_{t}(w) \log r(t)$, we obtain formula (5.6). Thus the proof is finished in this case.

Assume now that $\tilde{G}_{t}$ is given by (5.23). Then, on the one hand, by Lemma 5.12 the function $J(v):=\tilde{G}_{t}(i v+1 / 2), v \in \mathbb{R}$, should have a finite purely imaginary limit for $v \rightarrow+\infty$ or for $v \rightarrow-\infty$. On the other hand, from (5.21) it follows that $J(v)$ can be written as a periodic function of $v$ plus the linear term $-i r^{\prime}(t) v /(r(t) \log (t))$. Therefore, $r^{\prime}(t)=0$ and $J(v)$ is an imaginary constant. It follows that $G_{t}(z)=i C_{t} z$ for all $z \in \mathbb{A}_{r(t)}$ and some constant $C_{t} \in \mathbb{R}$. Note that $p \equiv 0$ belongs to $\mathcal{V}_{r}$ for any $r \in(0,1)$. So setting $p_{t}(w)=0$ for all $w \in D_{t}$ we again obtain formula (5.6). The proof is now complete.

Now we are going to establish some lemmas which will be used to prove sufficiency in Theorem [5.6. In what follows in this subsection we assume that $d \in[1,+\infty]$, $\left(D_{t}\right)$ is a non-degenerate canonical domain system of order $d$, and $\mathcal{D}:=\{(z, t): t \geq$ $\left.0, z \in D_{t}\right\}$.

Lemma 5.13. Let $r \in(0,1)$. Suppose $p \in \mathcal{V}_{r}$ is given by (5.4). Then for any $z \in \mathbb{A}_{r}$,

$$
\begin{aligned}
|p(z)| & \leq \mu_{1}(\mathbb{T})+\frac{2}{1-r}\left(\frac{|z|}{1-|z|}+\frac{r}{|z|-r}\right) \\
-\operatorname{Re} p(z) & \leq\left(\mathcal{K}_{r}(r /|z|)-1\right) \mu_{2}(\mathbb{T}) \leq \frac{4 r(1-\rho) \mu_{2}(\mathbb{T})}{(\rho-r)(1-r)^{2}}, \quad \rho:=|z| .
\end{aligned}
$$

Proof. Inequality $1-r^{2 k} \geq 1-r$, where $k \in \mathbb{N}$, and the Laurent expansion of $\mathcal{K}_{r}$ in $\mathbb{A}_{r}$,

$$
\mathcal{K}_{r}(z)=\frac{1+z}{1-z}+\sum_{k=1}^{+\infty} \frac{2 r^{2 k}}{1-r^{2 k}}\left(z^{k}-z^{-k}\right)=1+2 \sum_{k=1}^{+\infty}\left(\frac{z^{k}}{1-r^{2 k}}-\frac{r^{2 k} z^{-k}}{1-r^{2 k}}\right)
$$


allows us to estimate $\left|\mathcal{K}_{r}(z)\right|$ and $\left|1-\mathcal{K}_{r}(r / z)\right|$, which together with (5.4) leads to (5.24).

The inequality $\operatorname{Re} p(z) \geq\left(1-\mathcal{K}_{r}(r /|z|)\right) \mu_{2}(\mathbb{T})$ follows from (5.4) and Remark 5.2 . Then, again using (5.26), we obtain (5.25).

Lemma 5.14. Let $G: \mathcal{D} \rightarrow \mathbb{C}$. Suppose that there exist functions $p: \mathcal{D} \rightarrow \mathbb{C}$ and $C:[0,+\infty) \rightarrow \mathbb{R}$ such that conditions (i)-(iv) are fulfilled. Then $G$ is a weak holomorphic vector field of order $d$ in $\mathcal{D}$.

Proof. Conditions WHVF1 and WHVF2 from Definition 2.1 hold trivially.

To prove WHVF3, fix any compact $K \subset \mathcal{D}$. Then there exists $\delta>0$ and $T>0$ such that $r(t)+\delta \leq|z| \leq 1-\delta$ and $t \leq T$ for all $(z, t) \in K$. Applying Lemma 5.13 , and taking into account that $r(T) \leq r(t) \leq r(0)$ for all $t \in[0, T]$, from (5.24) we deduce that

$$
|G(z, t)| \leq|C(t)|+\frac{\left|r^{\prime}(t)\right|}{r(T)}\left(1+\frac{4 / \delta}{1-r(0)}\right) \quad \text { for all }(z, t) \in K
$$

Thus appealing to condition (iv) and Definition 4.1 completes the proof.

Proof of Theorem 5.6: Sufficiency. Suppose that there exist functions $p: \mathcal{D} \rightarrow$ $\mathbb{C}$ and $C:[0,+\infty) \rightarrow \mathbb{R}$ such that conditions (i)-(iv) are fulfilled. Then by Lemma 5.14 $G$ is a weak holomorphic vector field of order $d$ in $\mathcal{D}$. It remains to prove that it is semicomplete. Assume on the contrary that there exists $(z, s) \in \mathcal{D}$ such that the domain of definition $J_{*}(z, s)$ of the unique maximal solution $w_{s}^{*}(\cdot, z)$ to the initial value problem (2.1) (see Theorem 2.3) is bounded from above. Denote $t^{*}:=\sup J_{*}(z, s)$ and $\rho(t):=\left|w_{s}^{*}(t, z)\right|$ for all $t \in\left[s, t^{*}\right)$. Apply Lemma 5.13. According to (i) and (5.25),

$$
\rho^{\prime}(t)=\rho(t) \frac{r^{\prime}(t)}{r(t)} \operatorname{Re} p\left(w_{s}^{*}(t, z), t\right) \leq-\frac{4 r^{\prime}(t)(1-\rho(t))}{(\rho(t)-r(t))(1-r(t))^{2}} \quad \text { for a.e. } t \in\left[s, t^{*}\right) .
$$

We claim that $\rho(t) \leq \rho^{*}(t):=1-(1-\rho(s)) \exp \alpha(r(t)-r(s))$ for all $t \in$ $\left[s, t^{*}\right)$, where $\alpha:=4(\rho(s)-r(s))^{-1}(1-r(s))^{-2}$. Indeed, consider the function $f(t):=\rho(t)-\rho^{*}(t)$. This function is locally absolutely continuous in $\left[s, t^{*}\right)$. Since $\rho^{*}(t) \geq \rho(s)$ and $r(t) \leq r(s)$ for all $t \in\left[s, t^{*}\right)$, from (5.27) it follows that $f^{\prime}(t) \leq 0$ for a.e. $t \in\left[s, t^{*}\right)$ such that $f(t) \geq 0$. Bearing in mind that $f(s)=0$, we therefore conclude that $\rho(t)-\rho^{*}(t) \leq 0$ for all $t \in\left[s, t^{*}\right)$.

It follows that since $t^{*}<+\infty$, there exists $\delta_{1}>0$ such that $\rho(t)=\left|w_{s}^{*}(z, t)\right|<$ $1-\delta_{1}$ for all $t \in\left[s, t^{*}\right)$. By Remark [5.8, $\tilde{p}(z, t):=1-p(r(t) / z, t)$ belongs to $\mathcal{V}_{r(t)}$ for each $t \geq 0$. Therefore, choosing $\rho(t):=\left|r(t) / w_{s}^{*}(z, t)\right|$ in the above argument, one can also conclude that there exists $\delta_{2}>0$ such that $\left|w_{s}^{*}(z, t)\right|-r(t)>\delta_{2}$ for all $t \in\left[s, t^{*}\right)$. The fact that these conclusions contradict Theorem 2.3 (iii) proves that the vector field $G$ is semicomplete.

Proof of Theorem 5.6: Necessity. Suppose that $G$ is a semicomplete weak holomorphic vector field of order $d$. By Proposition 5.7 there exist functions $p: \mathcal{D} \rightarrow \mathbb{C}$ and $C:[0,+\infty) \rightarrow \mathbb{R}$ satisfying conditions (i) and (iii). It remains to prove (ii) and (iv). 
First of all, we notice that $\operatorname{Im} \mathcal{N}\left(p_{t}\right)=0$ for all $t \geq 0$ because $\mathcal{N}\left(\mathcal{K}_{r(t)}\right)=1$ (see Remark [5.5). Hence $C(t)=(1 / 2 \pi) \operatorname{Im} \int_{\mathbb{T}} G(\rho \xi, t) /(\rho \xi)|d \xi|$, where we have fixed some $\rho \in(r(0), 1)$. Since by definition $G(\cdot, z)$ is measurable for all $z \in \mathbb{A}_{r(0)}$ and for each $T>0$ there exists a non-negative $k_{T} \in L^{d}([0, T], \mathbb{R})$ such that $|G(z, t)| \leq k_{T}(t)$ whenever $|z|=\rho$ and $t \in[0, T]$, it follows with the help of the Lebesgue dominated convergence theorem that $t \mapsto C(t)$ belongs to $L_{\text {loc }}^{d}([0,+\infty), \mathbb{R})$. This proves (iv).

We are left with the proof of (ii). Fix any $s \geq 0$ and any $w \in D_{s}$. On one hand, by the construction we made in the proof of Proposition 5.7, $p(w, t)=0$ for a.e. $t \geq s$ such that $r^{\prime}(t)=0$. On the other hand, $t \mapsto p(w, t)$ is measurable in $E_{*}:=\left\{t \geq s: r^{\prime}(t) \neq 0\right\}$, because $t \mapsto r^{\prime}(t) p(t) / r(t)=G(w, t) / w-i C(t)$ is measurable and $t \mapsto r(t)$ is locally absolutely continuous in $[0,+\infty)$. Thus $t \mapsto p(w, t)$ is measurable in $[s,+\infty)$. Statement (ii) now follows easily.

5.2. Degenerate case. In this subsection we will show that if a canonical domain system $\left(D_{t}\right)$ is degenerate, i.e., $D_{t}:=\mathbb{D}^{*}:=\mathbb{D} \backslash\{0\}$ for all $t \geq 0$, then any evolution family $\left(\varphi_{s, t}\right)$ over $\left(D_{t}\right)$ can be extended to an evolution family in $\mathbb{D}$ with a common Denjoy-Wolff point at the origin. Namely, we prove the following

Proposition 5.15 (compare [1, Prop. (1.4.30)]). Let $d \in[1,+\infty]$. Suppose $D_{t}:=$ $\mathbb{D}^{*}$ for all $t \geq 0$, and let $\left(\varphi_{s, t}\right)$ be an $L^{d}$-evolution family over $\left(D_{t}\right)$. Then $\lim _{z \rightarrow 0} \varphi_{s, t}(z)=0$ for any $s \geq 0$ and $t \geq s$ and the formula

$$
\phi_{s, t}:= \begin{cases}\varphi_{s, t}(z), & \text { if } z \in \mathbb{D}^{*} \\ 0, & \text { if } z=0\end{cases}
$$

defines an $L^{d}$-evolution family in $\mathbb{D}$.

Proof. Fix any $s \geq 0$ and any $t \geq s$. Since $\varphi_{s, t}$ is bounded in $\mathbb{D}^{*}$, the origin is its removable singularity. By Lemma 4.8 $\varphi_{s, t} \in \mathbb{M}(0,0)$. Hence $\lim _{z \rightarrow 0} \varphi_{s, t}(z)=0$.

The fact that $\left(\phi_{s, t}\right)$ satisfies conditions EF1, EF2, and EF3 of Definition 3.1 follows from the corresponding conditions in Definition 4.3 except for EF3 with $z=0$, which holds by the mere fact that $\phi_{s, t}(0)=0$ for all $s \geq 0$ and all $t \geq s$. The proof is complete.

The converse statement is obvious: if $\left(\phi_{s, t}\right)$ is an $L^{d}$-evolution family in $\mathbb{D}$ and $\phi_{s, t}(0)=0$ for all $s \geq 0$ and all $t \geq s$, then $\left(\varphi_{s, t}\right):=\left(\left.\phi_{s, t}\right|_{\mathbb{D}^{*}}\right)$ is an $L^{d}$-evolution family over $\left(D_{t}\right)$ with $D_{t}=\mathbb{D}^{*}$ for all $t \geq 0$.

Taking into account Theorem 5.1 and the above Proposition 5.15] one can deduce from the results of [10] a constructive characterization of semicomplete weak holomorphic vector fields for the degenerate case. Indeed, on the one hand, 10, Theorem 1.1] establishes the one-to-one correspondence between Herglotz vector fields and evolution families in $\mathbb{D}$, while [10, Theorem 4.8] characterizes Herglotz vector fields in terms of the Berkson-Porta representation, see Remarks 3.4 and 3.5. On the other hand, Theorem 5.1 establishes the analogous one-to-one correspondence between evolution families and semicomplete weak holomorphic vector fields in the doubly connected settings. Hence, in view of Proposition 5.15, semicomplete weak holomorphic vector fields of order $d \in[1,+\infty]$ over the degenerate canonical domain system are exactly the Herglotz vector fields, given by [10, Theorem 4.8] with $\tau(t)=0$ for a.e. $t \in[0,+\infty)$. 
In this way we obtain the following analogue of Theorem 5.6 for the degenerate case. Let us recall that by $\mathcal{C}$ we denote the Carathéodory class of all functions $p \in \operatorname{Hol}(\mathbb{D}, \mathbb{C})$ such that $p(0)=1$ and $\operatorname{Re} p(w)>0$ for all $w \in \mathbb{D}$.

Proposition 5.16. Let $\left(D_{t}\right)$ be a degenerate canonical domain system. Then $G$ : $\mathcal{D} \rightarrow \mathbb{C}$, where $\mathcal{D}:=\left\{(z, t): t \geq 0, z \in D_{t}\right\}=\mathbb{D}^{*} \times[0,+\infty)$, is a semicomplete weak holomorphic vector field in $\mathcal{D}$ of order $d \in[1,+\infty]$ if and only if there exist functions $\alpha:[0,+\infty) \rightarrow[0,+\infty), C:[0,+\infty) \rightarrow \mathbb{R}$, and $p: \mathcal{D} \rightarrow \mathbb{C}$ satisfying the following conditions:

(i) $G(w, t)=w[i C(t)-\alpha(t) p(w, t)]$ for a.e. $t \geq 0$ and all $w \in D_{t}$;

(ii) for each $w \in D:=\bigcup_{t \geq 0} D_{t}$ the function $p(w, \cdot)$ is measurable in $E_{w}:=$ $\{t \geq 0:(w, t) \in \mathcal{D}\}$

(iii) for each $t \geq 0$ the function $p(\cdot, t)$ belongs to the Carathéodory class $\mathcal{C}$;

(iv) $C \in L_{\text {loc }}^{d}([0,+\infty), \mathbb{R})$ and $\alpha \in L_{\text {loc }}^{d}([0,+\infty),[0,+\infty))$.

\section{EXAMPLES}

Example 6.1. A set of trivial examples can be obtained by considering static non-degenerate canonical domain systems $\left(D_{t}\right)$, i.e., canonical domain systems for which $D_{t}$ does not depend on $t$ and does not coincide with the punctured disk $\mathbb{D}^{*}$, say $D_{t}:=\mathbb{A}_{r}$ for all $t \geq 0$ and some constant $r \in(0,1)$.

In this case by Theorem 5.6 the semicomplete weak holomorphic vector fields of order $d$ are exactly the functions of the form $G(w, t)=i C(t) w$, where $C$ belongs to $L_{\text {loc }}^{d}([0,+\infty), \mathbb{R})$. Hence, according to Theorem 5.1 , the $L^{d}$-evolution families $\left(\varphi_{s, t}\right)$ over static non-degenerate canonical domain systems are just families of rotations, $\varphi_{s, t}(z)=z e^{i(\theta(t)-\theta(s))}$, where $\theta \in A C^{d}([0,+\infty), \mathbb{R})$.

Example 6.2. According to the classical Denjoy-Wolff theorem, a self-mapping of the unit disk cannot have more than one fixed point unless it is the identity map. The infinitesimal version of this statement implies that a Herglotz vector field $G(z, t)$ in the unit disk (see Definition 3.3) cannot have more than one zero for almost every $t \geq 0$ such that $G(\cdot, t)$ does not vanish identically; see Remark 3.5 .

For mappings of the class $\mathbb{M}\left(r_{1}, r_{2}\right)$ the situation is different. One can have any finite number of fixed points in $\mathbb{A}_{r}$. Now we show an example of an evolution family over an $L^{\infty}$-canonical system of annuli sharing an arbitrary finite number of fixed points.

Let $N \in \mathbb{N}, r_{0} \in(0,1)$ and $r_{*} \in\left(r_{0}, 1\right)$. Take $r(t):=r_{0} e^{-t}$ and denote $R(t):=$ $r(t)^{N}, R_{*}:=r_{*}^{N}$,

$$
\alpha(t):=\frac{\mathcal{K}_{R(t)}\left(R(t) / R_{*}\right)-1}{\mathcal{K}_{R(t)}\left(R_{*}\right)+\mathcal{K}_{R(t)}\left(R(t) / R_{*}\right)-1} .
$$

According to Remark 5.2, $\alpha(t)$ is well defined for all $t \geq 0$ and satisfies $0<\alpha(t)<1$. Consider the two positive measures on $\mathbb{T}, \mu_{1}:=\alpha(t) \mu, \mu_{2}:=(1-\alpha(t)) \mu$, where

$$
\mu:=\frac{1}{N} \sum_{j=0}^{N-1} \delta_{2 \pi j / N}
$$

and $\delta_{\theta}$ denotes the Dirac measure with atom at the point $\xi=e^{i \theta}$. 
The corresponding function $p=p_{t} \in \mathcal{V}_{r(t)}$ given by representation (5.4) is

$$
\begin{aligned}
& p_{t}(z)=\frac{1}{N}\left(\alpha(t) \sum_{j=0}^{N-1} \mathcal{K}_{r(t)}\left(e^{-2 i \pi j / N} z\right)\right. \\
& \left.\quad+(1-\alpha(t)) \sum_{j=0}^{N-1}\left[1-\mathcal{K}_{r(t)}\left(e^{2 i \pi j / N} r(t) / z\right)\right]\right) .
\end{aligned}
$$

Define $F(z, c):=(1+c z) /(1-c z), c>0$. Decomposing $F\left((c z)^{N}, 1\right)$ into partial fractions, we get $\sum_{j=0}^{N-1} F\left(z e^{2 i \pi j / N}, c\right)=N F\left(z^{N}, c^{N}\right)$. It follows that $\sum_{j=0}^{N-1} \mathcal{K}_{r}\left(z e^{2 i \pi j / N}\right)=N \mathcal{K}_{r^{N}}\left(z^{N}\right)$ for all $r \in(0,1)$ and $z \in \mathbb{A}_{r}$. In particular, we have

$$
p_{t}(z)=\alpha(t) \mathcal{K}_{R(t)}\left(z^{N}\right)+(1-\alpha(t))\left[1-\mathcal{K}_{R(t)}\left(R(t) / z^{N}\right)\right] .
$$

On the one hand, by (6.1) and (6.2), $p_{t}\left(z_{j}\right)=0$ for all $t \geq 0$ and $j=0,1, \ldots, N-1$, where $z_{j}:=r_{*} e^{2 i \pi j / N}$. On the other hand, $p_{t} \in \mathcal{V}_{r(t)}$ for each $t \geq 0$ and $t \mapsto p_{t}(z)$ is smooth in $t$, for each fixed $z \in \mathbb{D}^{*}$.

By Theorem [5.6, it follows that $G(z, t):=w p_{t}(w)$ is an $L^{\infty}$-semicomplete weak holomorphic vector field over $\left(D_{t}\right)$ with $G\left(z_{j}, t\right)=0$ for all $j=0,1, \ldots, N-1$ and all $t \geq 0$. According to Theorem 5.1 this vector field generates an $L^{\infty}$-evolution family $\left(\varphi_{s, t}\right)$ over $\left(D_{t}\right)$ such that $\varphi_{s, t}\left(z_{j}\right)=z_{j}$ for all $j=0,1, \ldots, N-1$ and all $s$ and $t$ satisfying $0 \leq s \leq t$.

Example 6.3. Let $\left(\left(D_{t}\right),\left(\varphi_{s, t}\right)\right)$ be a non-degenerate evolution family of order $d \in$ $[1,+\infty]$. Define $\tilde{\varphi}_{s, t}(z):=r(t) / \varphi_{s, t}(r(s) / z)$ for all $s \geq 0$, all $t \geq s$ and all $z \in D_{s}$. Then it is easy to deduce from Theorem 4.4 that $\left(\tilde{\varphi}_{s, t}\right)$ is also an $L^{d}$-evolution family over $\left(D_{t}\right)$. According to Theorem 5.6 the semicomplete weak holomorphic vector field $G$ corresponding to $\left(\varphi_{s, t}\right)$ is given by $G(w, t)=w\left[i C(t)+r^{\prime}(t) p(w, t) / r(t)\right]$ for a.e. $t \geq 0$ and all $w \in D_{t}$, where the functions $C$ and $p$ are subject to conditions (ii)(iv) from this theorem. Then the vector field corresponding to the evolution family $\left(\tilde{\varphi}_{s, t}\right)$ is given by $\tilde{G}(w, t)=w\left[-i C(t)+r^{\prime}(r) \tilde{p}(w, t) / r(t)\right]$, where by Remark [5.8,

$$
\tilde{p}(w, t):=1-p(r(t) / w)=p\left[r(t), \widehat{\mu_{2}^{t}}, \widehat{\mu_{1}^{t}}\right](w) \quad \text { for all } t \geq 0 \text { and all } w \in D_{t},
$$

and the families $\left(\mu_{1}^{t}\right)_{t \geq 0}$ and $\left(\mu_{2}^{t}\right)_{t \geq 0}$ are defined by $p(\cdot, t)=p\left[r(t), \mu_{1}^{t}, \mu_{2}^{t}\right]$ for all $t \geq 0$.

\section{COMMENTS ON PARAMETRIC REPRESENTATION OF SLIT MAPPINGS}

Let $0<m<1<M<+\infty$ and $A:=\{\zeta: m<|\zeta|<M\}$. Denote by $\mathbb{U}(A)$ the class of all univalent holomorphic functions $f: A \rightarrow \mathbb{C}^{*}$ such that $f(1)=1$ and for any closed curve $\gamma \in A$ the index $I(f \circ \gamma)$ of the origin w.r.t. the curve $f \circ \gamma$ coincides with $I(\gamma)$.

The following theorem, generalizing results of Komatu 25], Goluzin [21, and Li En Pir 34 on slit mappings of annuli, is due to Lebedev [33] 3

Theorem 7.1 (Lebedev). The following statements hold:

\footnotetext{
${ }^{3}$ Note that the paper 33 is a short communication, so it does not contain any proofs.
} 
(A) Let $f \in \mathbb{U}(A)$. Suppose that $\partial f(A)$ consists of two disjoint open Jordan curves one of which extends to $\infty$ and the other ends at the origin. Then for any function $\lambda:[0,+\infty) \rightarrow[0,1]$, continuous in $[0,+\infty)$ except for a finite number of jump discontinuities and subject to the condition $\int_{0}^{+\infty} \lambda(t) d t=\int_{0}^{+\infty}(1-\lambda(t)) d t=+\infty$, there exist continuous functions $\kappa_{j}:[0,+\infty) \rightarrow \mathbb{T}, j=1,2$, such that

$$
f(\zeta)=\lim _{t \rightarrow+\infty} f(\zeta, t) \quad \text { for all } \zeta \in A,
$$

where $f=f(\zeta, t)$ is the solution to the initial value problem

$$
\begin{aligned}
& \frac{\dot{f}}{f}=\lambda(t)\left[\mathcal{K}_{r(t)}\left(\kappa_{1}(t) m_{t} / f\right)-\mathcal{K}_{r(t)}\left(\kappa_{1}(t) m_{t}\right)\right] \\
& -(1-\lambda(t))\left[\mathcal{K}_{r(t)}\left(\kappa_{2}(t)^{-1} r(t) f / m_{t}\right)-\mathcal{K}_{r(t)}\left(\kappa_{2}(t)^{-1} r(t) / m_{t}\right)\right],\left.\quad f\right|_{t=0}=\zeta, \\
& r(t):=e^{-t} m / M, \text { and } t \mapsto m_{t} \text { is the solution to } \\
& \begin{aligned}
\frac{\dot{m}_{t}}{m_{t}} & =-\lambda(t) \operatorname{Re} \mathcal{K}_{r(t)}\left(\kappa_{1}(t) m_{t}\right) \\
& \quad(1-\lambda(t))\left[1-\operatorname{Re} \mathcal{K}_{r(t)}\left(\kappa_{2}(t)^{-1} r(t) / m_{t}\right)\right],\left.\quad m_{t}\right|_{t=0}=m .
\end{aligned}
\end{aligned}
$$

(B) Given any functions $\lambda:[0,+\infty) \rightarrow[0,1]$ and $\kappa_{j}:[0,+\infty) \rightarrow \mathbb{T}$, $j=1,2$, continuous in $[0,+\infty)$ except for a finite number of jump discontinuities, the problem (7.1) (7.2) has a unique solution $f(\zeta, t)$ defined for all $t \geq 0$ and all $\zeta \in A$, with $f(\cdot, t) \in \mathbb{U}(A)$ for any $t \geq 0$.

(C) Under the conditions of $(B), f(\cdot, t)$ converges in $A$ as $t \rightarrow+\infty$ to some function $f \in \mathbb{U}(A)$.

In this section we would like to explain how the dynamics of system (7.1)-(7.2) is connected to the evolution families we consider in this paper.

First of all, take $r(t):=e^{-t} m / M$, and let $D_{t}:=\mathbb{A}_{r(t)}$ for all $t \geq 0$. Clearly, $\left(D_{t}\right)$ is a non-degenerate canonical domain system of order $d=\infty$. Since from (7.2) it follows that $(d / d t) \log m_{t} \leq \mathcal{K}_{r(t)}\left(r(t) / m_{t}\right)-1$ and $(d / d t) \log \left(r(t) / m_{t}\right) \leq \mathcal{K}_{r(t)}\left(m_{t}\right)-1$ whenever solution $t \mapsto m_{t}$ to (7.2) exists, we can conclude, arguing in the same way as in the proof of the sufficiency statement of Theorem 5.6 stated previously, that for any measurable functions $\lambda:[0,+\infty) \rightarrow[0,1]$ and $\kappa_{j}:[0,+\infty) \rightarrow \mathbb{T}$, $j=1,2$, the initial value problem has a unique solution $[0,+\infty) \ni t \mapsto m_{t}$ and that $r(t)<m_{t}<1$ for all $t \geq 0$. Now with the change of variables $z:=\zeta / M$, $w:=r(t) f / m_{t}$, the initial value problem (7.1) is equivalent to

$$
\begin{aligned}
& \dot{w}=G(w, t),\left.\quad w\right|_{t=0}=z, \quad \text { where } \quad G(w, t):=w[i C(t)-p(w, t)], \\
& C(t):=(1-\lambda(t)) \operatorname{Im} \mathcal{K}_{r(t)}\left(\kappa_{2}(t)^{-1} r(t) / m_{t}\right)-\lambda(t) \operatorname{Im} \mathcal{K}_{r(t)}\left(m_{t} \kappa_{1}(t)\right), \\
& p(\cdot, t):=p\left[r(t),(1-\lambda(t)) \nu_{2}^{t}, \lambda(t) \nu_{1}^{t}\right] \quad \text { for all } t \geq 0 \text { and all } w \in D_{t},
\end{aligned}
$$

and $\nu_{j}^{t}, j=1,2, t \geq 0$, stands for the Dirac measure on $\mathbb{T}$ with the atom at $\kappa_{j}(t)$.

It is easy to see that functions $C$ and $p$ satisfy conditions (ii)-(iv) from Theorem 5.6 with $d=+\infty$. Hence $G$ is a semicomplete weak holomorphic vector field of order $+\infty$ and $f(\zeta, t)=m_{t} \varphi_{0, t}(\zeta / M) / r(t)$ for all $t \geq 0$, where $\left(\varphi_{s, t}\right)$ is the $L^{\infty}$-evolution family over $\left(\mathbb{A}_{r(t)}\right)$ generated by $G$ in the sense of Theorem [5.1(iv). 
Therefore we conclude that dynamics of system (7.1) - 7.2 can be regarded as a very special case of dynamics of evolution families we consider in this paper and that statement (B) of Lebedev's theorem follows from our results. In particular, equation (5.1) with the vector field $G$ admitting the representation given in Theorem [5.6 is a generalization of the Komatu equation [25, 21] (known also as the Goluzin-Komatu equation), since (7.1)-(7.2) reduces to the latter equation when $\lambda \equiv 0$ and $m=1$.

\section{REFERENCES}

[1] M. Abate, Iteration theory of holomorphic maps on taut manifolds, Mediterranean, Rende, 1989. MR1098711 (92i:32032)

[2] M. Abate, F. Bracci, M.D. Contreras, and S. Díaz-Madrigal, The evolution of Loewner's differential equation. To appear in Newsletter of the European Mathematical Society, 2010, issue 78, 31-38. MR2768999

[3] N. I. Akhiezer, Elements of the theory of elliptic functions, translated from the second Russian edition by H. H. McFaden, Translations of Mathematical Monographs, 79, Amer. Math. Soc., Providence, RI, 1990. MR1054205 (91k:33016)

[4] I. A. Aleksandrov, Parametric continuations in the theory of univalent functions (in Russian), Izdat. "Nauka", Moscow, 1976. MR0480952 (58:1099)

[5] L. Arosio, F. Bracci, H. Hamada, G. Kohr, An abstract approach to Loewner's chains, to appear in J. Anal. Math. Available on ArXiv 1002.4262

[6] R. O. Bauer and R. M. Friedrich, Stochastic Loewner evolution in multiply connected domains, C. R. Math. Acad. Sci. Paris 339 (2004), no. 8, 579-584. MR2111355

[7] R. O. Bauer and R. M. Friedrich, On radial stochastic Loewner evolution in multiply connected domains, J. Funct. Anal. 237 (2006), no. 2, 565-588. MR2230350 (2007d:60007)

[8] R. O. Bauer and R. M. Friedrich, On chordal and bilateral SLE in multiply connected domains, Math. Z. 258 (2008), no. 2, 241-265. MR2357634(2009b:60292)

[9] E. Berkson and H. Porta, Semigroups of analytic functions and composition operators, Michigan Math. J. 25 (1978), no. 1, 101-115. MR0480965 (58:1112)

[10] F. Bracci, M. D. Contreras, and S. Díaz-Madrigal, Evolution families and the Loewner equation I: the unit disk, to appear in J. Reine Angew. Math. Available on ArXiv 0807.1594. DOI: 10.1515/crelle.2011.167.

[11] F. Bracci, M. D. Contreras, and S. Díaz-Madrigal, Evolution families and the Loewner equation II: complex hyperbolic manifolds, Math. Ann. 344 (2009), 947-962. MR.2507634 (2010f:32020)

[12] F. Bracci, M. D. Contreras, and S. Díaz-Madrigal, Semigroups versus evolution families in the Loewner theory, J. Anal. Math. 115 (2011), 273-292. MR2855040

[13] L. de Branges, A proof of the Bieberbach conjecture, Acta Math. 154 (1985), no. 1-2, 137152. MR0772434 (86h:30026)

[14] T. Casavecchia, S. Díaz-Madrigal, A non-autonomous version of the Denjoy-Wolff theorem, to appear in Complex Anal. Oper. Theory. Avaliable on ArXiv 0910.2875. DOI: 10.1007/s11785-011-0214-6.

[15] E. A. Coddington and N. Levinson, Theory of ordinary differential equations, McGraw-Hill Book Company, Inc., New York, 1955. MR0069338 (16,1022b)

[16] M. D. Contreras, S. Díaz-Madrigal, and P. Gumenyuk, Loewner chains in the unit disk. Revista Matemática Iberoamericana 26 (2010), 975-1012. MR2789373

[17] M. D. Contreras, S. Díaz-Madrigal, and P. Gumenyuk, Geometry behind chordal Loewner chains, Complex Anal. Oper. Theory 4 (2010), 541-587 MR2719792(2011h:30037)

[18] P. L. Duren, Univalent functions, Grundlehren der Mathematischen Wissenschaften, 259, Springer, New York, 1983. MR0708494 (85j:30034)

[19] A. F. Filippov, Differential equations with discontinuous right-hand sides (in Russian), "Nauka", Moscow, 1985. MR0790682 (87f:34002) translation to English: Kluwer Acad. Publ., Dordrecht, 1988. MR 1028776 (90i:34002)

[20] C. H. FitzGerald and Ch. Pommerenke, The de Branges theorem on univalent functions, Trans. Amer. Math. Soc. 290 (1985), 683-690. MR792819 (87b:30023) 
[21] G. M. Goluzin, On the parametric representation of functions univalent in a ring, (in Russian) Mat. Sbornik N.S. 29(71) (1951), 469-476. MR0047780(13,930b)

[22] V. Ja. Gutljanskiı̌, Parametric representation of univalent functions (in Russian), Dokl. Akad. Nauk SSSR 194 (1970), 750-753. MR0271324 (42:6207) English translation in Soviet Math. Dokl. 11 (1970), 1273-1276.

[23] V. Ya. Gutljanskŭ, Parametric representations and extremal problems in the theory of univalent functions (in Russian), Dissertation Dokt. Fiz.-Mat. Nauk, Mat. Inst. Akad. Nauk Ukrain. SSR, Kiev, 1972,

[24] F. Holland, The extreme points of a class of functions with positive real part, Math. Ann. 202 (1973), 85-87. MR0335782(49:562)

[25] Y. Komatu, Untersuchungen über konforme Abbildung von zweifach zusammenhängenden Gebieten (in German), Proc. Phys.-Math. Soc. Japan (3) 25 (1943), 1-42. MR0016129 $(7,514 \mathrm{e})$ Avaliable via Journal@rchive, http://www.journalarchive.jst.go.jp

[26] Y. Komatu, Darstellungen der in einem Kreisringe analytischen Funktionen nebst den Anwendungen auf konforme Abbildung über Polygonalringgebiete, Jap. J. Math. 19 (1945), 203-215. MR0014449 (7,287b)

[27] Y. Komatu, On conformal slit mapping of multiply-connected domains, Proc. Japan Acad. 26 (1950), no. 7, 26-31. MR0046437(13,734c)

[28] P. P. Kufarev, On one-parameter families of analytic functions (in Russian. English summary), Rec. Math. [Mat. Sbornik] N.S. 13 (55) (1943), 87-118. MR0013800 (7:201g)

[29] P. P. Kufarev, V. V. Sobolev, and L. V. Sporyševa, A certain method of investigation of extremal problems for functions that are univalent in the half-plane (in Russian), Trudy Tomsk. Gos. Univ. Ser. Meh.-Mat. 200 (1968), 142-164. MR0257336 (41:1987)

[30] J. Kurzweil, Ordinary differential equations, translated from the Czech by Michal Basch, Studies in Applied Mechanics, 13, Elsevier, Amsterdam, 1986. MR0929466 (88m:34001)

[31] M. P. Kuvaev and P. P. Kufarev, An equation of Löwner's type for multiply connected regions (in Russian), Tomskiǔ Gos. Univ. Uč. Zap. Mat. Meh. 25 (1955), 19-34. MR.0087734(19,401f)

[32] G. F. Lawler, Conformal invariance and 2D statistical physics, Bull. Amer. Math. Soc. (N.S.) 46 (2009), no. 1, 35-54. MR2457071 (2010a:60283)

[33] N. A. Lebedev, On parametric representation of functions regular and univalent in a ring (in Russian), Dokl. Akad. Nauk SSSR (N.S.) 103 (1955), 767-768. MR0072950 (17,356f)

[34] E. P. Li, On the theory of univalent functions on a circular ring (in Russian), Doklady Akad. Nauk SSSR (N.S.) 92 (1953), 475-477. MR.0059357 (15,516d)

[35] K. Löwner, Untersuchungen über schlichte konforme Abbildungen des Einheitskreises (in German), Math. Ann. 89 (1923), 103-121. MR1512136

[36] I. Markina and A. Vasil'ev, Virasoro algebra and dynamics in the space of univalent function, in Five Lectures in Complex Analysis (Sevilla, Spain, 2008), 85-116, Contemp. Math., 525, Amer. Math. Soc., Providence, RI, 2010. MR2683220 (2011i:81189)

[37] Ch. Pommerenke, Über die subordination analytischer funktionen (in German), J. Reine Angew Math. 218 (1965), 159-173. MR0180669 (31:4900)

[38] Ch. Pommerenke, Univalent functions. With a chapter on quadratic differentials by Gerd Jensen, Vandenhoeck \& Ruprecht, Göttingen, 1975. MR0507768 (58:22526)

[39] G. Sansone, Equazioni Differenziali nel Campo Reale, Vol. 2 (in Italian), Nicola Zanichelli, Bologna, 1949. MR0030663(11,32a)

[40] O. Schramm, Scaling limits of loop-erased random walks and uniform spanning trees, Israel J. Math. 118 (2000), 221-288. MR1776084 (2001m:60227)

[41] D. Shoikhet, Semigroups in geometrical function theory, Kluwer Acad. Publ., Dordrecht, 2001. MR1849612 (2002g:30012)

[42] J. Tsai, Loewner chains on the universal covering space of a Riemann surface, Preprint 2008. Avaliable on ArXiv 0812.3721

[43] T. Vaitsiakhovich, Boundary value problems for complex partial differential equations in a ring domain, Ph.D. Thesis, Freie Universität Berlin, 2008. Available on http://www.diss.fuberlin.de, http://www.diss.fu-berlin.de/diss/receive/FUDISS_thesis_000000003859.

[44] G. Valiron, Fonctions analytiques (in French), Presses Univ. France, Paris, 1954. MR0061658 (15:861a)

[45] D. Zhan, Stochastic Loewner evolution in doubly connected domains, Probab. Theory Related Fields 129 (2004), no. 3, 340-380. MR2128237 (2006b:82111) 
[46] V. A. Zmorovič, On certain classes of analytic functions in a circular ring (in Russian), Mat. Sb. N.S. 40(82) (1956), 225-238. MR0083042(18,648h)

Camino de los Descubrimientos, s/n, Departamento de Matemática Aplicada II, EsCuela Técnica Superior de Ingeniería, Universidad de Sevilla, Sevilla, 41092, Spain

E-mail address: contreras@us.es

Camino de los Descubrimientos, s/n, Departamento de Matemática Aplicada II, Escuela Técnica Superior de Ingeniería, Universidad de Sevilla, Sevilla, 41092, Spain

E-mail address: madrigal@us.es

Department of Mathematics, University of Bergen, Johannes Brunsgate 12, Bergen 5008, NORWAY

E-mail address: Pavel.Gumenyuk@math.uib.no

Current address: Dipartimento di Matematica, Università di Roma "Tor Vergata", Via della Ricerca Scientifica, 1. 00133 Roma, Italy

E-mail address: gumenyuk@axp.mat.uniroma2.it 